\title{
Time-Frequency Fault Feature Extraction for Rolling Bearing Based on the Tensor Manifold Method
}

\author{
Fengtao Wang, ${ }^{1}$ Shouhai Chen, ${ }^{1}$ Jian Sun, ${ }^{1}$ Dawen Yan, ${ }^{2}$ Lei Wang, ${ }^{1}$ and Lihua Zhang ${ }^{3}$ \\ ${ }^{1}$ Institute of Vibration Engineering, School of Mechanical Engineering, Dalian University of Technology, Dalian 116024, China \\ ${ }^{2}$ School of Mathematical Sciences, Dalian University of Technology, Dalian 116024, China \\ ${ }^{3}$ Institute of Microelectromechanical Systems and Precision Engineering, School of Mechanical Engineering, \\ Dalian University of Technology, Dalian 116024, China
}

Correspondence should be addressed to Fengtao Wang; wangft@dlut.edu.cn

Received 2 May 2014; Revised 20 June 2014; Accepted 10 July 2014; Published 4 August 2014

Academic Editor: Weihua Li

Copyright (C) 2014 Fengtao Wang et al. This is an open access article distributed under the Creative Commons Attribution License, which permits unrestricted use, distribution, and reproduction in any medium, provided the original work is properly cited.

Rolling-bearing faults can be effectively reflected using time-frequency characteristics. However, there are inevitable interference and redundancy components in the conventional time-frequency characteristics. Therefore, it is critical to extract the sensitive parameters that reflect the rolling-bearing state from the time-frequency characteristics to accurately classify rolling-bearing faults. Thus, a new tensor manifold method is proposed. First, we apply the Hilbert-Huang transform (HHT) to rolling-bearing vibration signals to obtain the HHT time-frequency spectrum, which can be transformed into the HHT time-frequency energy histogram. Then, the tensor manifold time-frequency energy histogram is extracted from the traditional HHT time-frequency spectrum using the tensor manifold method. Five time-frequency characteristic parameters are defined to quantitatively depict the failure characteristics. Finally, the tensor manifold time-frequency characteristic parameters and probabilistic neural network (PNN) are combined to effectively classify the rolling-bearing failure samples. Engineering data are used to validate the proposed method. Compared with traditional HHT time-frequency characteristic parameters, the information redundancy of the time-frequency characteristics is greatly reduced using the tensor manifold time-frequency characteristic parameters and different rolling-bearing fault states are more effectively distinguished when combined with the PNN.

\section{Introduction}

Rolling bearings are widely used in modern rotating machinery, and their failure is one of the most common causes of machine breakdowns and accidents [1-3]. Therefore, fault diagnosis of rolling bearings is necessary to ensure the safe and efficient operation of machines in engineering applications. The main aspects of bearing fault diagnosis are classification and pattern recognition, where feature extraction directly affects the accuracy and reliability of the fault diagnosis [4]. Rolling-bearing fault features can be generally divided into three categories: time-domain characteristics, frequency-domain characteristics, and time- and frequencydomain characteristics $[5,6]$.

Time-domain characteristics are fairly intuitive; however, they fluctuate significantly and lack quantitative judging criteria. Thus, they cannot be directly used to diagnose bearing faults. In contrast, frequency-domain characteristics can be used to diagnose bearing fault conditions more accurately because different bearing faults correspond to different characteristic frequencies. However, there are typically noise and modulation components in the bearing fault signals. Thus, direct application of the frequency-domain method will submerge the fault characteristic frequency in noise or false frequency components because of improper selection of the demodulation parameters. Furthermore, signal denoising and demodulation must be conducted before extracting the bearing fault characteristic frequencies. In the process of signal denoising and demodulation, parameters such as the denoising parameters, demodulation center, and filter bandwidth should be properly selected based on experience, and a satisfactory selection is only obtained after numerous adjustments.

The time- and frequency-domain characteristics, which have the intuitive feature of the time-domain characteristics 
and good time-frequency aggregation, can simultaneously reflect the time-domain and frequency-domain characteristics of a signal [7-10]. Therefore, extracting the timefrequency fault characteristics is important for fault diagnosis. Wang and $\mathrm{Hu}$ used the principle of time-frequency image analysis to diagnose gearbox faults in 1993 [11]; this effort was the first application of time-frequency image for the fault diagnosis of machinery and equipment. Zhang et al. subsequently used time-frequency images to classify diesel engine faults under complex vibration conditions [12]. Zhu et al. used short-time Fourier transform to extract timefrequency features for fault diagnosis [13], and satisfactory results were achieved. However, the aforementioned timefrequency characteristics are not adaptive and can only be used for reciprocating machinery. To overcome the limitations of the above methods, Huang et al. proposed the HHT time-frequency spectrum, which is self-adaptive [14]. The HHT time-frequency spectrum is suitable for analyzing nonstationary signals because of its frequency instantaneity [15]. However, mode mixing is inevitable for signals with instantaneous frequency trajectory crossings $[16,17]$. Li et al. used the geometric center of the HHT time-frequency spectrum as a feature vector $[18,19]$ in combination with SVM and classified rolling-bearing fault signals. However, because the geometric center requires a considerable amount of calculations and lacks corresponding physical meaning, it can only provide qualitative classification criteria. Manifold learning has recently emerged in nonlinear-feature extraction because of its capability of effectively identifying hidden low-dimensional nonlinear structures in high-dimensional data. He [20] proposed a time-frequency manifold feature by combining the time-frequency distribution and the nonlinear manifold for an effective quantitative representation of machinery health pattern.

This paper proposes a new tensor manifold timefrequency feature extraction method to overcome the weakness of traditional HHT time-frequency characteristics. The HHT time-frequency spectrum, which contains a considerable amount of failure information, is used as the research object. The tensor manifold learning method is applied to extract the tensor manifold time-frequency characteristics of the HHT time-frequency spectrum. The two-dimensional time-frequency information does not need to be converted into a one-dimensional vector when calculating the tensor manifold, and the information loss is significantly reduced. On this basis, five time-frequency characteristic parameters are defined. The tensor manifold time-frequency characteristic parameters can distinguish different rolling-bearing fault states more effectively than traditional HHT timefrequency characteristic parameters. Combined with PNN, the tensor manifold time-frequency characteristic parameters can effectively distinguish different rolling-bearing fault states. Engineering vibration signals were used to evaluate the efficiency of the proposed method.

The remainder of this paper is organized as follows. The theory basis is introduced in Section 2, and the tensor manifold time-frequency fault feature extraction method is described in Section 3. Section 4 presents the adaption of the proposed method to rolling-bearing fault classification. Rolling-bearing fault classification is implemented in Section 5. Finally, conclusions are drawn in Section 6.

\section{Theory Basis}

2.1. HHT Time-Frequency Spectrum. Based on the definition of instantaneous frequency and EMD, the HHT timefrequency spectrum is analytically derived as follows.

Apply the EMD to signal $X(t)$ to obtain the IMFs of $X(t)$. Then, the analytical form of $X(t)$ can be expressed as

$$
X(t)=\operatorname{Re} \sum_{i=1}^{n} A_{i}(t) \mathrm{e}^{j \int \omega_{i}(t) d t},
$$

where Re is the real part of the selected signal, $A_{i}(t)$ is the instantaneous amplitude of the $i$ th IMF, and $\omega_{i}(t)$ is the corresponding instantaneous frequency.

The time, frequency, and amplitude of the signals can be combined to form the three-dimensional time-frequency space. Then, the amplitude distribution on time-frequency plane is referred to as the HHT time-frequency spectrum, which is expressed as

$$
H(t, \omega)=\operatorname{Re} \sum_{i=1}^{n} b_{i} A_{i}(t) \mathrm{e}^{j \int \omega_{i}(t) d t},
$$

where Re is the real part of the selected signal and $b_{i}$ is the indicator variable. When $\omega_{i}=\omega, b_{i}=1$, and when $\omega_{i} \neq \omega$, $b_{i}=0$.

The HHT time-frequency analysis is a decomposition method based on signal local characteristics, which provides a physical basis for the concept of instantaneous frequency and sets this method apart from conventional methods through its use of numerous harmonic components to describe complex nonlinear and nonstationary signals. Therefore, from the concept definition and the nature of signal analysis, the HHT time-frequency spectrum eliminates the limitations of Fourier transform and can accurately describe nonstationary signal characteristics.

\subsection{Tensor Manifold Algorithm}

2.2.1. Locality Preserving Projection (LPP) Manifold Learning Algorithm. The LPP manifold learning algorithm aims at finding the linear transformation matrix $\mathbf{W}$ to reduce the dimensionality of high-dimensional data. There are $l$ training samples $\left\{\mathbf{x}_{i}\right\}_{i=1}^{l} \in \mathbf{R}^{m}$, and $\mathbf{W}$ can be obtained by minimizing the following objective function:

$$
\min _{\mathbf{W}}\left(\sum_{i, j}\left(\mathbf{W}^{T} \mathbf{x}_{i}-\mathbf{W}^{T} \mathbf{x}_{j}\right)^{2} S_{i, j}\right),
$$

where $S_{i, j}$ is the similarity measure among objects and can be defined using the $k$-nearest-neighbor method:

$$
S_{i, j}=\left\{\exp \left(-\frac{\left\|\mathbf{x}_{i}-\mathbf{x}_{j}\right\|_{f}^{2}}{t}\right), \text { if } \mathbf{x}_{i} \in O\left(k, \mathbf{x}_{i}\right) \text { or } \mathbf{x}_{j} \in O\left(k, \mathbf{x}_{j}\right)\right\},
$$


where $O\left(k, \mathbf{x}_{i}\right)$ denotes the $k$ nearest neighbor of $\mathbf{x}_{i}$ and $t$ is a positive constant. Both $k$ and $t$ can be determined empirically.

Equation (3) demonstrates the feature space after dimension reduction can maintain the local structure of the original high-dimensional space. We apply an algebraic transformation to (3) as follows:

$$
\begin{aligned}
& \frac{1}{2} \sum_{i, j}\left(\mathbf{W}^{T} \mathbf{x}_{i}-\mathbf{W}^{T} \mathbf{x}_{j}\right)^{2} S_{i j} \\
& \quad=\sum_{i, j} \mathbf{W}^{T} \mathbf{x}_{i} D_{i i} \mathbf{x}_{i}^{T} \mathbf{W}-\sum_{i, j} \mathbf{W}^{T} \mathbf{x}_{i} S_{i j} \mathbf{x}_{i}^{T} \mathbf{W} \\
& \quad=\mathbf{W}^{T} \mathbf{X}(\mathbf{D}-\mathbf{S}) \mathbf{X}^{T} \mathbf{W}=\mathbf{W}^{T} \mathbf{X} \mathbf{L} \mathbf{X}^{T} \mathbf{W},
\end{aligned}
$$

where $\mathbf{X}=\left[\mathbf{x}_{1}, \mathbf{x}_{2}, \ldots, \mathbf{x}_{l}\right], \mathbf{D}$ denotes an $l \times l$ diagonal matrix, where the diagonal element $D_{i i}=\sum_{i} S_{i j}, \mathbf{S}=\left(S_{i j}\right)_{l \times l}$, and $\mathbf{L}=\mathbf{D}-\mathbf{S}$.

Then, the problem of solving for the optimal vector $\mathbf{W}$ can be transformed into the following eigenvalue problem:

$$
\mathbf{X} \mathbf{L} \mathbf{X}^{T} \mathbf{W}=\lambda \mathbf{X D} \mathbf{X}^{T} \mathbf{W}
$$

2.2.2. Tensor LPP Manifold Learning Algorithm. The LPP manifold learning algorithm [21] can only be regarded as a one-dimensional manifold feature extraction algorithm. However, the number of training images in the twodimensional (e.g., time-frequency spectrum) image feature extraction process is notably small compared to the dimensions of the image vectors, which results in a singularity of $\mathbf{X D X}^{T}$ and failure of the LPP algorithm. To alleviate the drawback of the LPP, this paper uses a new tensor LPP manifold learning algorithm (Ten-LoPP) [22] to extract the time-frequency spectrum fault characteristics.

There are $l$ two-dimensional training images $\left\{\mathbf{A}_{i}\right\}_{i=1}^{l} \in$ $\mathbf{R}^{m \times n}$, where $\boldsymbol{\omega}$ denotes an $n$-dimensional unitization column vector. The main objective of the tensor manifold algorithm is to make each $m \times n$ image matrix $\mathbf{A}_{i}$ project onto $\boldsymbol{\omega}$ using a linear transformation $\mathbf{y}_{i}=\mathbf{A}_{i} \boldsymbol{\omega}$. In this manner, an $m$ dimensional column vector can be obtained and considered a projection feature vector of image $\mathbf{A}_{i}$. The objective function of the tensor manifold algorithm is expressed as follows:

$$
\begin{aligned}
& \frac{1}{2} \sum_{i, j}\left(\mathbf{A}_{i} \boldsymbol{\omega}-\mathbf{A}_{j} \boldsymbol{\omega}\right)^{2} S_{i j} \\
& \quad=\sum_{i, j} \mathbf{W}^{T} \mathbf{A}_{i}^{T} D_{i i} \mathbf{I}_{m} \mathbf{A}_{i} \boldsymbol{\omega}-\sum_{i, j} \mathbf{W}^{T} \mathbf{A}_{i}^{T} S_{i j} \mathbf{I}_{m} \mathbf{A}_{i} \boldsymbol{\omega} \\
& \quad=\mathbf{W}^{T} \mathbf{A}^{T}\left[(\mathbf{D}-\mathbf{S}) \otimes \mathbf{I}_{m}\right] \mathbf{A W}=\mathbf{W}^{T} \mathbf{A}^{T}\left(\mathbf{L} \otimes \mathbf{I}_{m}\right) \mathbf{A W},
\end{aligned}
$$

where $\mathbf{A}=\left[\mathbf{A}_{1}, \mathbf{A}_{2}, \ldots, \mathbf{A}_{1}\right]$, the definitions of $\mathbf{D}$ and $\mathbf{L}$ are identical to those in the LPP manifold learning method, and $\otimes$ denotes the Kronecker product.

Then, the problem of solving for the optimal vector $\boldsymbol{\omega}$ is transformed into the following eigenvalue problem:

$$
\mathbf{A}^{T}\left(\mathbf{L} \otimes \mathbf{I}_{m}\right) \mathbf{A} \boldsymbol{\omega}=\lambda \mathbf{A}^{T}\left(\mathbf{D} \otimes \mathbf{I}_{m}\right) \mathbf{A} \boldsymbol{\omega},
$$

where $\boldsymbol{\omega}$ is comprised of $d$ feature vectors that correspond to the smallest nonzero eigenvalues; that is, there are $d$ optimal projection vectors $\boldsymbol{\omega}$, which can form the projection matrix $\mathbf{W}=\left[\boldsymbol{\omega}_{1}, \boldsymbol{\omega}_{2}, \ldots, \boldsymbol{\omega}_{d}\right]$. For any image $\mathbf{A}_{x}$, there is

$$
\mathbf{y}_{x i}=\mathbf{A}_{x} \boldsymbol{\omega}_{i}, \quad i=1,2, \ldots, d,
$$

where $\mathbf{y}_{x 1}, \mathbf{y}_{x 2}, \ldots, \mathbf{y}_{x d}$ are the projection feature vectors of the sample image $\mathbf{A}_{x}$ and $\mathbf{y}_{x}=\left[\mathbf{y}_{x 1}, \mathbf{y}_{x 2}, \ldots, \mathbf{y}_{x d}\right]$, which is comprised of projection feature vectors, is the characteristic matrix of the sample image $\mathbf{A}_{x}$.

2.3. Probabilistic Neural Network (PNN). The neural composition structure and elements of the PNN are shown in Figure 1

In the PNN, characteristic parameters were transported into each node on the pattern layer through the input layer. Then, we apply layer nonlinear mapping to the input parameters in each node of the PNN pattern and complete the comparison between an unknown type with a known type. Finally, the characteristic parameters that represent the types are input to the next layer for processing. The node structure of the layers is used to be called the RBF center, and the node output is expressed as follows:

$$
\mathbf{O}_{i}=\mathbf{R}_{i}\left(\left\|\mathbf{X}-\boldsymbol{\omega}_{i}\right\|\right),
$$

where the $i$ th center vector is $\omega_{i}$, which is the same size as the input vector. $R_{i}(\cdot)$ denotes the radial basis function, which is typically a Gaussian function; that is,

$$
\exp \left(-\frac{\left\|\mathbf{X}-\boldsymbol{\omega}_{i}\right\|^{2}}{2 \sigma_{i}^{2}}\right),
$$

where $\sigma_{i}$ denotes the shape parameter that corresponds to the $i$ th component of the radial basis function.

To facilitate the calculation, $\mathbf{X}$ and $\boldsymbol{\omega}_{i}$ are processed with mathematical regularization and unit. Assume that $\mathbf{z}_{i}=\mathbf{X} \cdot \boldsymbol{\omega}_{i}$. Then, the above expression is expressed as follows:

$$
g\left(\mathbf{z}_{i}\right)=\exp \left[\frac{\left(\mathbf{z}_{i}-1\right)}{\sigma^{2}}\right] .
$$

Finally, through the output layer (decision-making layer), the characteristic parameters, which are derived from the pattern layer, are accumulated to provide the category feature vector, which is

$$
f_{A}(\mathbf{X})=\sum_{j=1}^{N} g\left(\mathbf{z}_{i}\right) .
$$

The PNN has the following characteristics: (1) the training convergence speed is high, making the PNN suitable for the real-time processing of various data types; (2) the pattern unit can form any nonlinear mapping judgment surface, which is closest to the optimal judgment surface bayes; (3) the selection of the RBF center kernel function has diversity, and the form of the kernel function has a small effect on the recognition results; and (4) the number of neuron nodes in each PNN layer is relatively stable, the hardware processing is convenient, and the fault tolerance is high. The PNN has been widely used in pattern recognition, prediction estimation, and filtering denoising. 


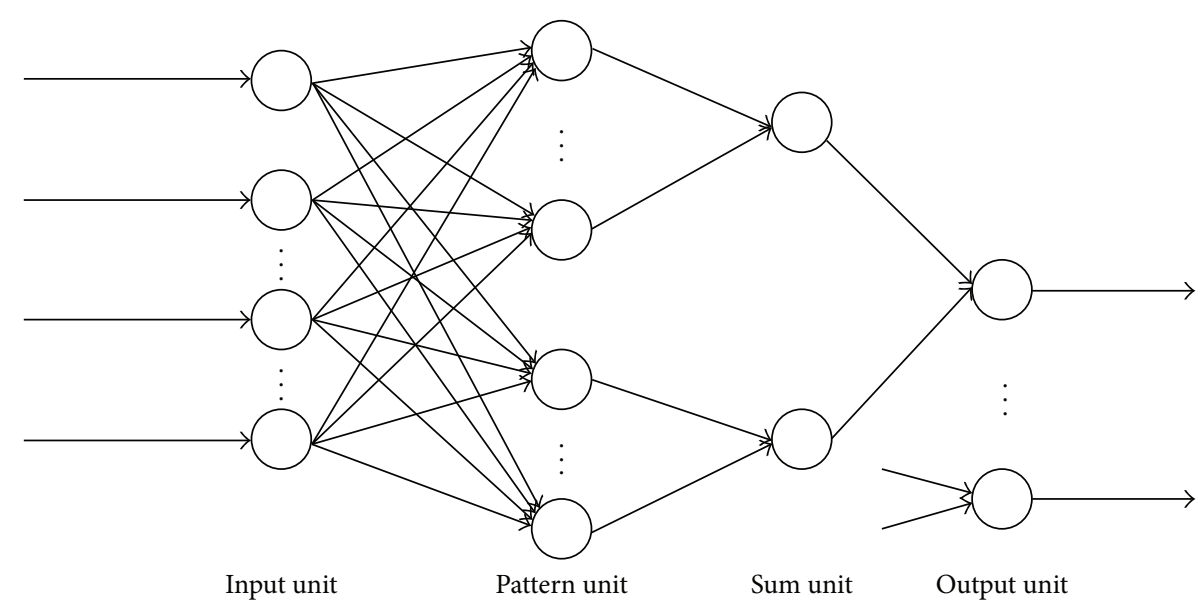

(a)

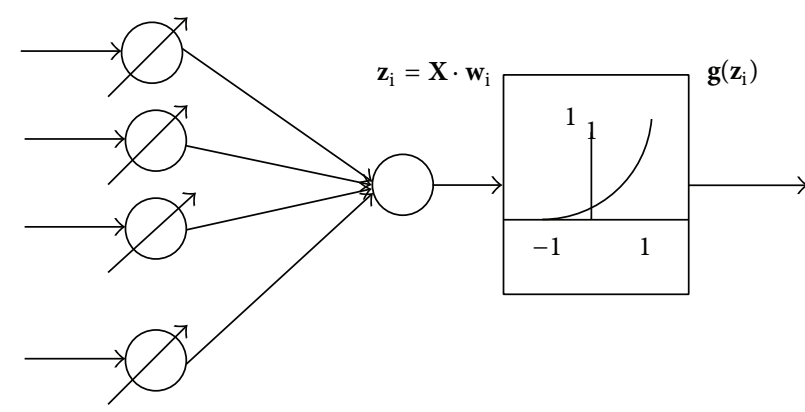

(b)

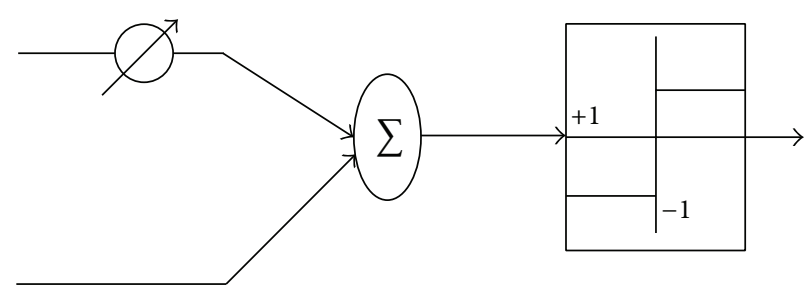

(c)

FIGURE 1: Internal composition structure and elements of the PNN: (a) system structure of the PNN, (b) pattern unit of the PNN, and (c) output unit of the PNN.

\section{Tensor Manifold Time-Frequency Fault Feature Extraction Method}

3.1. Description of the Proposed Method. The manifold learning method is a nonlinear dimension reduction method to extract low-dimensional nonlinear characteristics from highdimensional data. Unlike the conventional linear dimension reduction methods, such as multidimensional scaling (MDS), principal component analysis (PCA), and linear discriminant analysis (LDA), this method is a nonlinear method to address the part before the whole. By satisfying the entire optimization, the manifold learning method can preserve the partial manifold characteristics and effectively extract the nonlinear manifold characteristics that are inherent in the high-dimensional characteristic set. However, the manifold learning algorithm suffers from information loss and error that are caused by the transformation from a set of two-dimensional time-frequency characteristics to a onedimensional vector.

To alleviate the drawback of information loss and error, this section presents a tensor manifold time-frequency fault feature extraction method based on the tensor manifold algorithm to extract the set of low-dimensional timefrequency characteristics from the set of high-dimensional time-frequency characteristics. Then, five tensor manifold time-frequency characteristic parameters were defined and combined with the PNN to classify the rolling-bearing failure samples.

The tensor manifold time-frequency fault feature extraction method is described as follows, and Figure 2 presents its flow chart.

(1) Group the rolling-bearing vibration signal samples to be classified and for training and then calculate the HHT time-frequency spectrum. To hasten the calculation of the tensor manifold algorithm, grid the time-frequency regions, integrate the energy value of the HHT time-frequency spectrum of each mesh, and convert the HHT time-frequency spectrum into HHT time-frequency energy histograms.

(2) The HHT time-frequency energy histograms are essentially two-dimensional matrices. Use the HHT time-frequency energy histograms that correspond to signal samples to form a set of high-dimensional time-frequency characteristics.

(3) Apply the tensor manifold algorithm to extract the set of low-dimensional time-frequency characteristics from the set of high-dimensional time-frequency characteristics. In this manner, the tensor manifold time-frequency energy histograms are obtained.

(4) Based on the result of step (3), define different tensor manifold time-frequency characteristic parameters. 


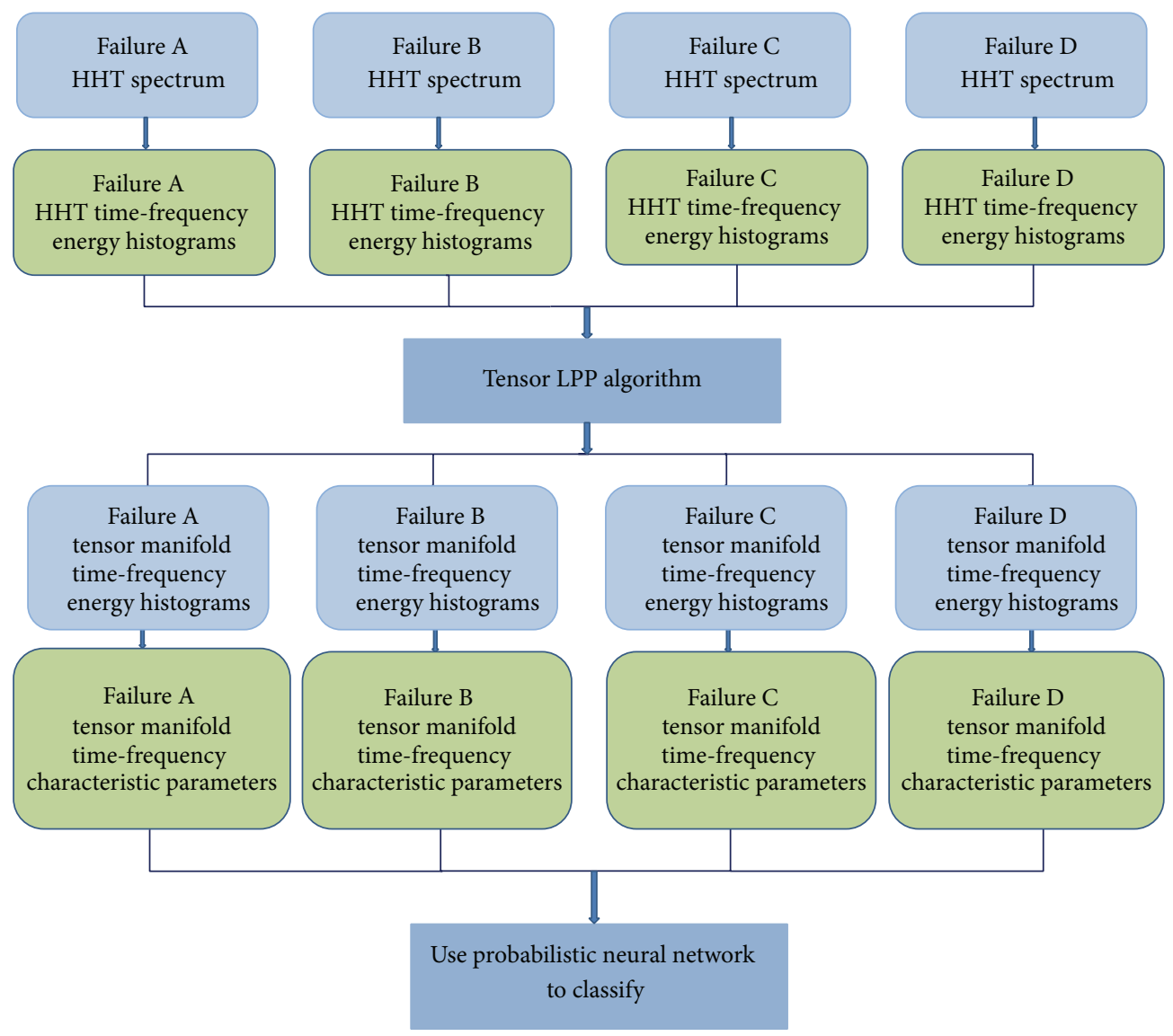

FIGURE 2: Flow chart of the time-frequency characteristic extraction method based on a tensor manifold.

Input the defined parameters of the training signal samples into the PNN for the rolling-bearing fault classification.

(5) Input the tensor manifold time-frequency characteristic parameters of the to-be-classified signal samples into the trained PNN to classify the rolling-bearing faults.

\subsection{Definition of the Time-Frequency Characteristic Parame-} ters. The tensor manifold time-frequency energy histogram is a nonlinear time-frequency fault feature and can effectively differentiate different rolling-bearing fault signals. However, it is equal to a two-dimensional matrix, which makes it unsuitable for direct application in fault classification. In this section, several parameters are presented to quantitatively measure the difference among the tensor manifold timefrequency energy histograms. Their definitions are provided as follows.

3.2.1. Energy Entropy. Entropy is proposed to measure the data complexity and the probability to generate the new signal model. Here, the energy entropy is defined as follows:

$$
H=-\sum_{i=1}^{n} p_{i} \log \left(p_{i}\right), \quad p_{i}=\frac{e_{i}}{\sum_{i=1}^{n} e_{i}}
$$

where $H$ is the energy entropy, $e_{i}$ is the value of the time frequency energy histogram, and $p_{i}$ is the proportion of each $e_{i}$ in the total $\sum_{i=1}^{n} e_{i}$. In addition, the energy entropy can reflect the uncertainty in the energy distribution.

3.2.2. Energy Correlation Coefficient. Divide the timefrequency energy histogram into $m$ sections by frequency and mark $\mathbf{E}_{f 1}, \mathbf{E}_{f 2}, \ldots, \mathbf{E}_{f m}$ and $\mathbf{E}_{t}=\mathbf{E}_{f 1}+\mathbf{E}_{f 2}+\cdots+\mathbf{E}_{f m}$. Because each $\mathbf{E}_{f i}$ varies with different time-frequency energy histograms, we can analyze the relevance of $\mathbf{E}_{f i}$ and $\mathbf{E}_{t}$ to measure the difference in the time-frequency energy histogram. The energy correlation coefficient vector is defined as follows:

$$
\text { Ecoef }=[\operatorname{corcoef}(1), \operatorname{corcoef}(2), \cdots \operatorname{corcoef}(m)]^{T},
$$

where $\operatorname{corcoef}(i)=\operatorname{corcoef}\left(\mathbf{E}_{f i}, \mathbf{E}_{t}\right)$ and $\operatorname{corcoef}(\cdot)$ is the cross-correlation function.

3.2.3. Energy Sparsity. The signal energy distribution of the time-frequency energy histogram varies more significantly as it approaches zero. The sparsity expresses the sparse distribution of energy, and the purpose of estimating the sparsity is to obtain a function $q(\mathbf{x}), \mathbf{x} \in \mathbf{R}^{n}$. If $\mathbf{x}$ is sparse, then $q(\mathbf{x})$ is relatively large, and vice versa. Generally, the norm $L_{p}$ of vector $\mathbf{x}$ is used to quantitatively estimate the sparsity. Here, 
we define the $L_{p}$ norm of the standardized form of vector $\mathbf{x}$ as follows:

$$
q^{-1}(\mathbf{x})=\frac{\|\mathbf{x}\|_{p}}{n^{1 / p-1 / 2} \cdot\|\mathbf{x}\|_{2}}=\frac{1}{n^{1 / p-1 / 2}} \cdot \frac{\left(\sum_{k=1}^{n} \mathbf{x}_{k}^{p}\right)^{1 / p}}{\left(\sum_{k=1}^{n} \mathbf{x}_{k}^{2}\right)^{1 / 2}}
$$

where $1 \leq p<\infty$, and we select $p=1$ such that $L_{1}$ can accurately reflect the energy distribution of the histogram.

3.2.4. Energy Mutual Information. Mutual information is proposed to measure the degree of independence among random variables. The mutual information of multiple variables is defined as the $K L$ divergence of the multivariate joint probability density and its marginal probability density product:

$$
\begin{aligned}
I(x) & =K L\left(p(x), \prod_{i=1}^{N} p_{i}\left(x_{i}\right)\right) \\
& =\int p(x) \log \left(\frac{p(x)}{\prod_{i=1}^{N} p_{i}\left(x_{i}\right)}\right) d x,
\end{aligned}
$$

where $K L$ is the divergence, $x=x_{1}, x_{2}, \ldots x_{N}, p(x)$ is the multivariate joint probability density function, and $p_{i}\left(x_{i}\right),(i=1 \sim N)$ is the marginal probability density function. Then $K L$ is defined as follows:

$$
K L[p(x), q(x)]=\int p(x) \log \frac{p(x)}{q(x)} d x,
$$

where $p(x), q(x)$ are two different probability density functions of a random vector $x$. The energy mutual information can be calculated for each histogram according to (18).

3.2.5. Energy Kurtosis. Kurtosis is a physical parameter that is proposed to measure the degree of Gaussian distribution of a random variable. A larger energy kurtosis in the time-frequency energy histogram corresponds to weaker Gaussianity of the energy distribution, whereas a smaller kurtosis indicates stronger Gaussianity. If the Gaussianity of the energy distribution is strong, the energy distribution presents the "middle big, two sides small" phenomenon. The energy values are mainly within the middle range, and larger or smaller values are less likely to occur. For the sequence $\mathbf{X}=x_{1}, x_{2}, \ldots x_{N}$ of energy values, the overall kurtosis is defined as

$$
\operatorname{Kurt}\left(x_{1,2, \ldots, N}\right)=\frac{\sum_{i=1}^{N}\left(x_{i}-\mu\right) / N}{\sigma^{4}}-3 .
$$

\section{Application of the Proposed Method to Rolling-Bearing Fault Classification}

4.1. Rolling-Bearing Fault Data. To verify the effectiveness of the proposed method, the new method was used to analyze bearing fault data from the Bearing Data Center of Case Western Reserve University [23].

The test rig, which is shown in Figure 3, was constructed for the run-to-failure testing of the rolling bearing. A $1.5 \mathrm{~kW}$

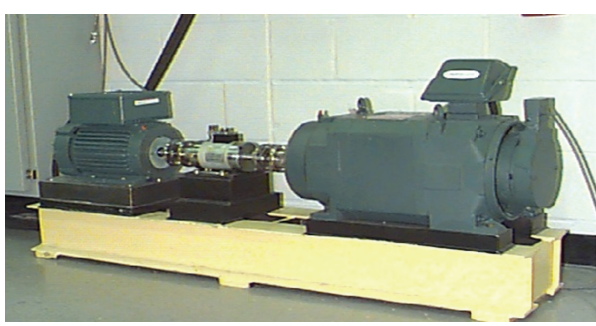

FIGURE 3: Diagram of the experimental test rig.

3-phase induction motor was connected to a power meter and torque sensor by self-calibration coupling, which drove the fan. The load was adjusted using the fan. Data were collected using a vibration acceleration sensor, which was vertically fixed above the chassis of the drive end bearings of the induction motor. The bearings are deep-groove ball bearings of the type SKF6205-2RS JEM. There is a single point of failure in the inner ball and outer surface of the machining spark; the failure sizes are $0.18 \mathrm{~mm}$ in diameter and $0.28 \mathrm{~mm}$ deep. The experimental data were collected with a sample frequency of $12,000 \mathrm{~Hz}$ and a shaft running speed of $29.53 \mathrm{~Hz}(1,772 \mathrm{rpm})$. The corresponding ball pass frequency inner-race (BPFI), ball rotation frequency (BS), and ball pass frequency outer-race (BPFO) were estimated to be $159.93,139.19$, and $105.87 \mathrm{~Hz}$, respectively.

Figures 4(a)-4(d) depict a group of normal, inner-race fault, ball fault, and outer-race fault time-domain signals. Although there are several differences among the four types of signals in the time-domain wave nature, it is difficult to distinguish the rolling-bearing fault conditions using these intuitive qualitative differences. Therefore, the fault features that quantitatively represent the differences of different rolling-bearing fault statuses must be studied.

\subsection{HHT Time-Frequency Characteristics of Rolling Bearings.} First, the HHT time-frequency spectra of four states were calculated using the HHT method. Then, the HHT timefrequency spectrum was divided into 64 regions of identical size. The histogram of the HHT time-frequency spectrum was obtained via the integral of the energy amplitude for each region. Different types of signal HHT time-frequency spectra and their histograms are shown in Figure 5.

Figure 5 describes the HHT time-frequency spectrum and corresponding time-frequency energy histograms of the normal rolling-bearing vibration signal. As shown in Figure 5, the time-frequency energy is mainly distributed in the low-frequency region and decreases with increasing frequency. The amplitude ranges from 0 to $20 \mathrm{~g}^{2}$.

Figure 6 presents the HHT time-frequency spectrum and corresponding time-frequency energy histogram with an inner-race fault. As shown in Figure 6, the time-frequency energy is widely distributed in both the low- and highfrequency regions, with a lull in the mid-frequency region. The amplitude ranges from 0 to $40 \mathrm{~g}^{2}$, the maximum value of which is greater than that in the normal case.

Figure 7 presents the HHT time-frequency spectrum and corresponding time-frequency energy histograms with a ball 


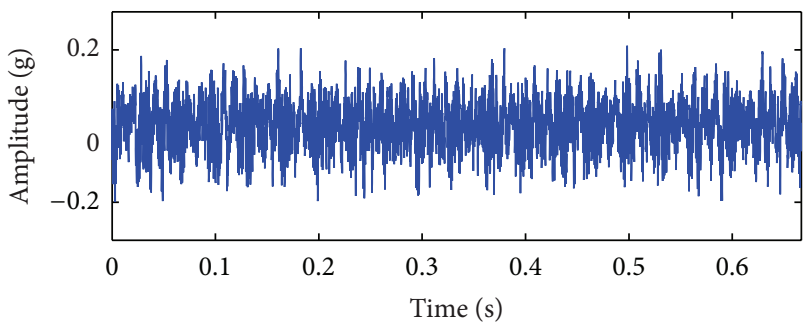

(a)

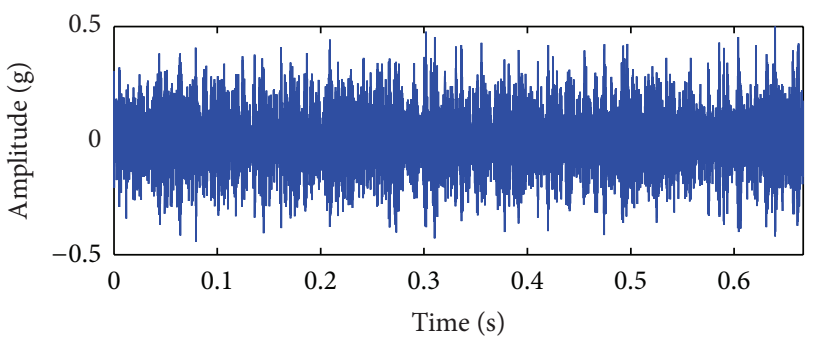

(c)

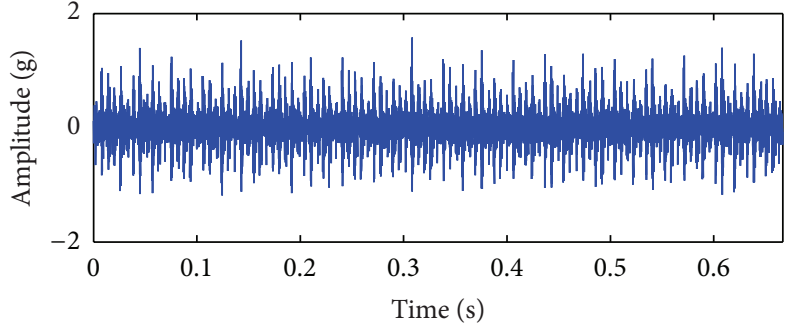

(b)

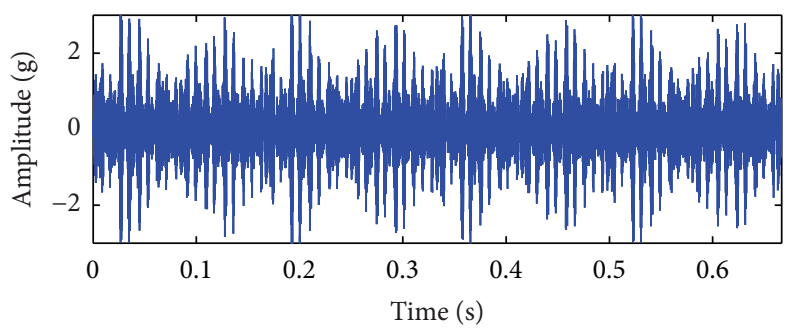

(d)

FIGURE 4: Four heterogeneous rolling-bearing fault data: (a) normal, (b) inner-race faults, (c) ball faults, and (d) outer-race faults.

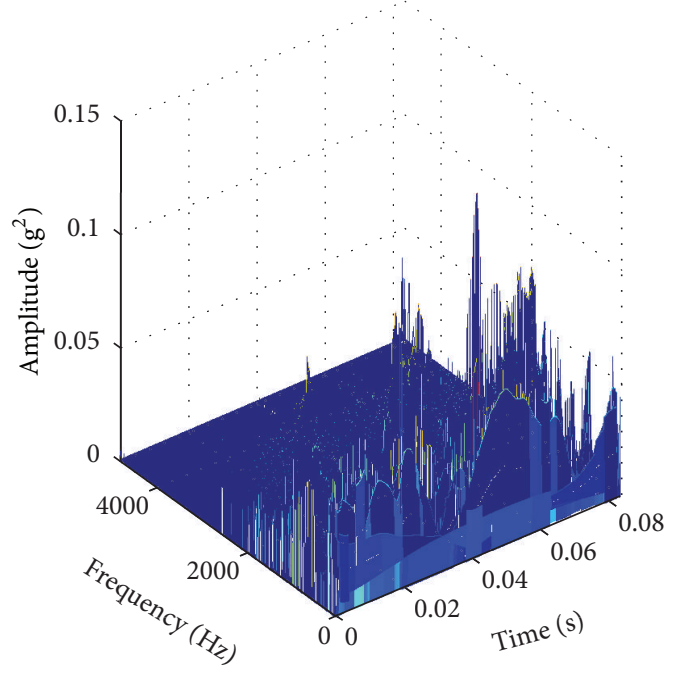

(a)

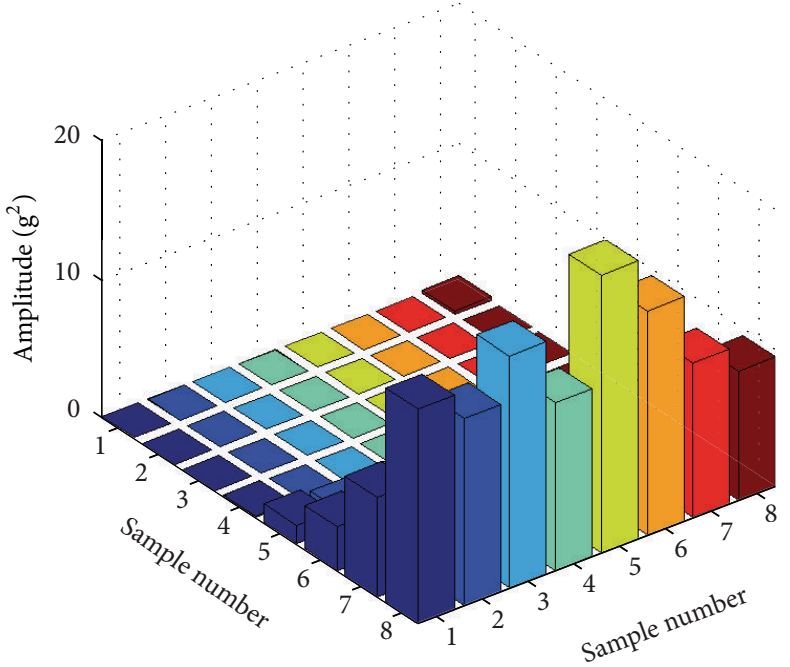

(b)

FIGURE 5: (a) Normal HHT time-frequency spectrum and (b) normal time-frequency energy histogram.

fault. As shown in Figure 7, the time-frequency energy is mainly distributed in the high-frequency region and exhibits a less significant yet stable distribution in the low-frequency region. The amplitude of the energy histogram ranges from 0 to $30 \mathrm{~g}^{2}$.

Figure 8 presents the HHT time-frequency spectrum and corresponding time-frequency energy histograms with an outer-race fault. As shown in Figure 8, the time-frequency energy is centered in the high-frequency region and exhibits a lull in the low-frequency region. The distribution trend begins at a rather low frequency and increases abruptly at a certain high frequency. The magnitude ranges from 0 to $100 \mathrm{~g}^{2}$.
4.3. Extraction of the Tensor Manifold Time-Frequency Characteristic Parameters. For convenience and conciseness, we only discuss the tensor manifold time-frequency characteristic parameters of four types of rolling-bearing faults in this section; in other situations, such as different damage degrees in the same fault type or different fault types with different damage degrees, the classification result will also be discussed in subsequent Section 5.

We consider 20 normal inner-race fault, ball fault, and outer-race fault time-domain signals (each sample dataset has 1,024 points, and 80 samples are used for training). The HHT time-frequency spectrum and HHT time-frequency energy histogram of each signal are obtained using the 


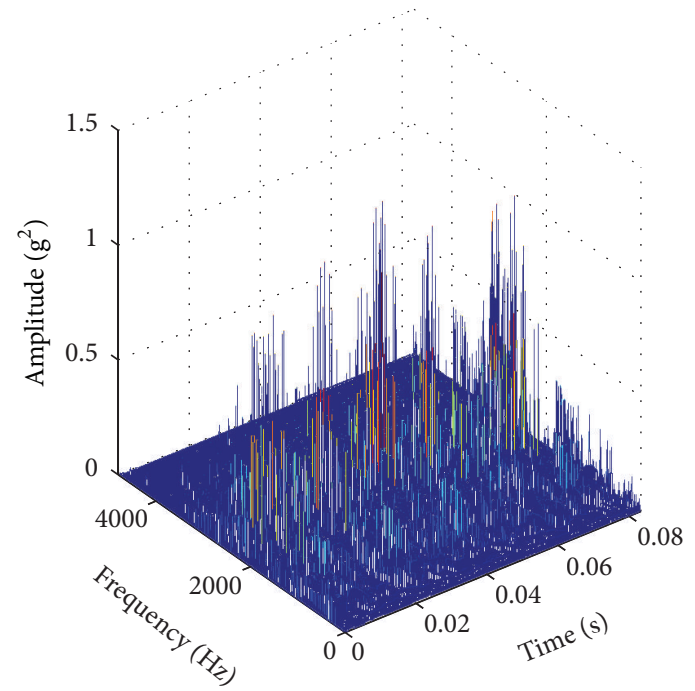

(a)

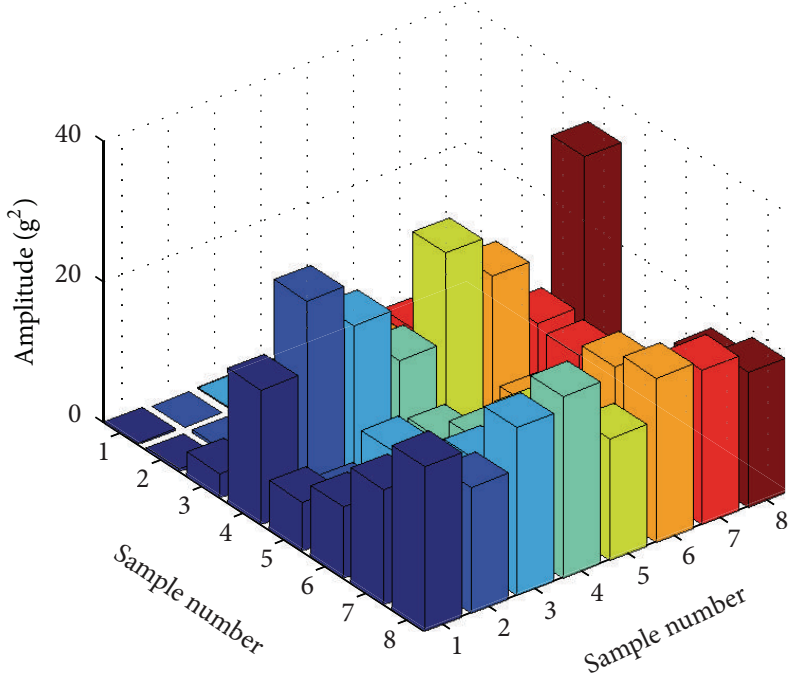

(b)

FIGURE 6: (a) HHT time-frequency spectrum with an inner-race fault and (b) time-frequency energy histogram with an inner-race fault.

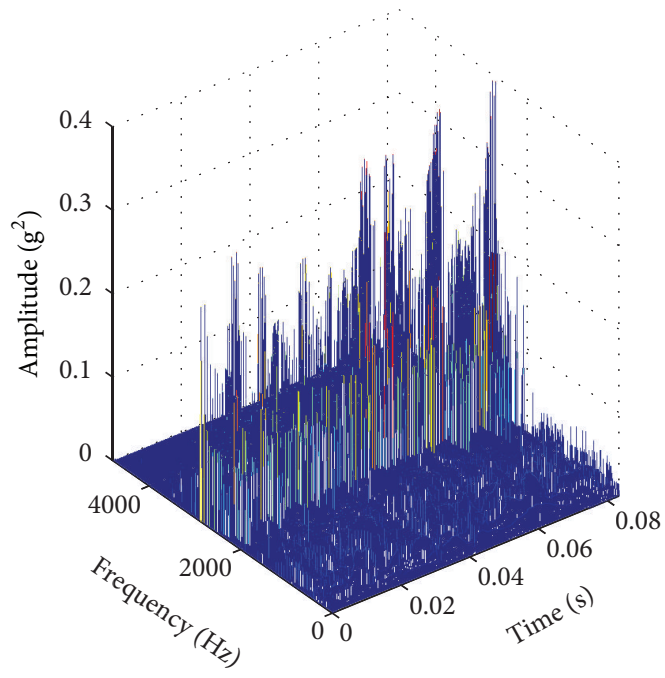

(a)

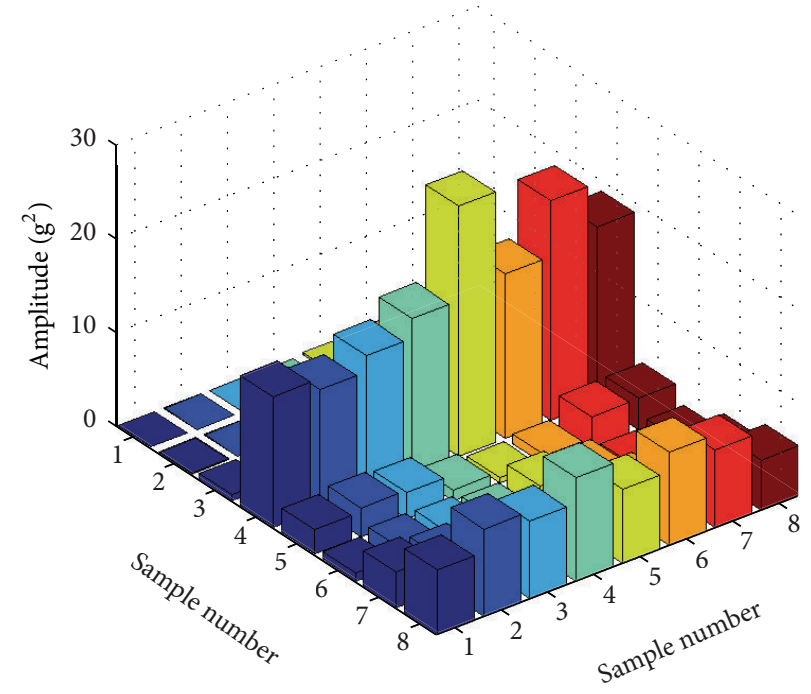

(b)

FIGURE 7: (a) HHT time-frequency spectrum with a ball fault and (b) time-frequency energy histogram with a ball fault.

aforementioned method. Then, a high-dimensional timefrequency feature combination with 80 samples is obtained. The tensor manifold algorithm is applied to extract the low-dimensional tensor manifold features from the highdimensional characteristic set. The optimal projected vectors $\mathbf{W}=\left[\boldsymbol{\omega}_{1}, \boldsymbol{\omega}_{2}, \ldots, \boldsymbol{\omega}_{d}\right]$ are obtained, and the parameter is defined as 6 because of the distribution of eigenvalues. Finally, we project the $8 * 8$ matrix energy histogram onto $\mathbf{W}$ and obtain the $8 * 6$ tensor manifold energy histograms.

According to the aforementioned definition of the five time-frequency characteristic parameters, we take the absolute value of the elements of the obtained $8 * 6$ tensor manifold energy histograms and calculate the tensor manifold timefrequency characteristic parameters of the tensor manifold energy histograms.
Five tensor manifold time-frequency characteristic parameters of the above 80 samples are obtained. Below, only 10 samples of the above four different types of rolling-bearing signals are considered for clarity in the graphics.

The results are as follows.

4.3.1. Manifold Energy Entropy. Samples 1-10 correspond to the normal signals, samples 11-20 correspond to the innerrace fault signals, samples 21-30 correspond to the ball fault signals, and samples $31-40$ correspond to the outer-race fault signals. The manifold energy entropy of each tensor manifold time-frequency energy histogram is calculated and depicted in Figure 9(a). The energy entropy of the HHT time-frequency energy histogram without a tensor manifold analysis is presented in Figure 9(b). Compared to Figure 9(a), 


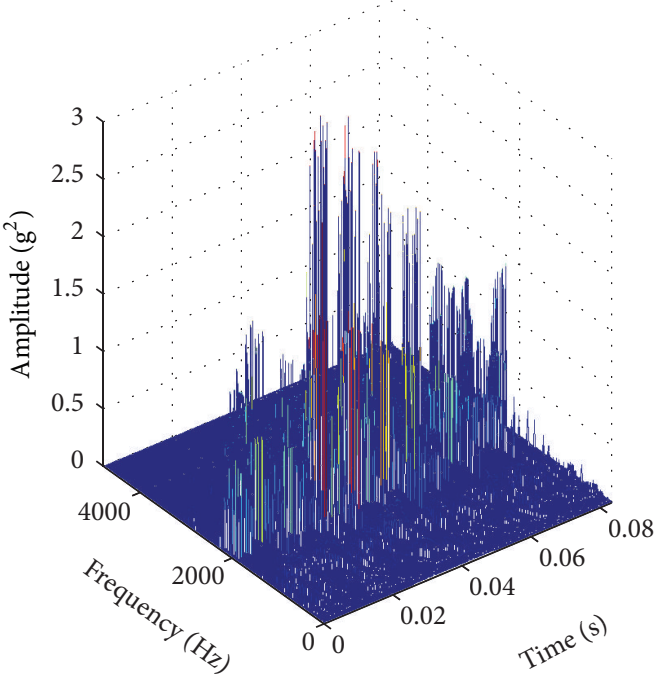

(a)

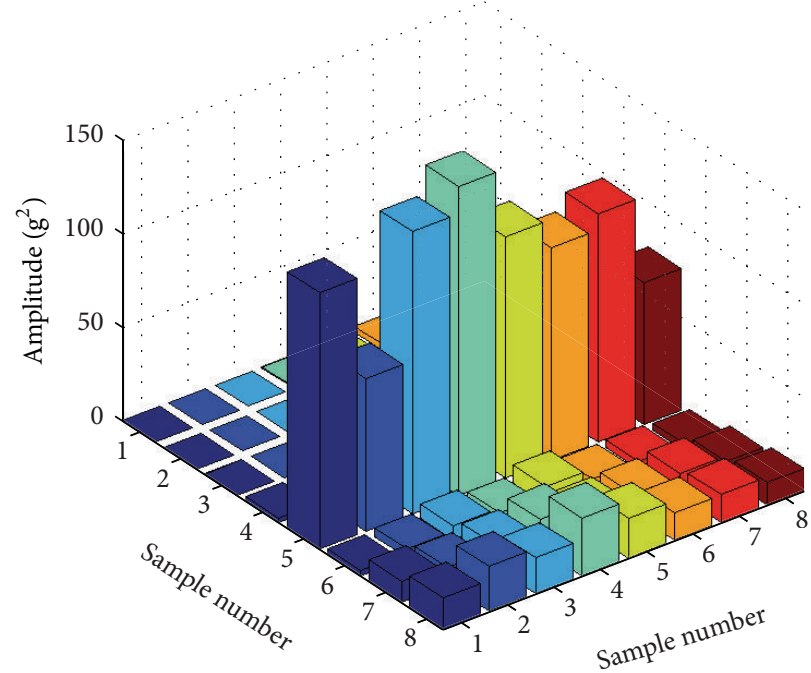

(b)

FIGURE 8: (a) HHT time-frequency spectrum with outer-race fault and (b) time-frequency energy histogram with outer-race fault.

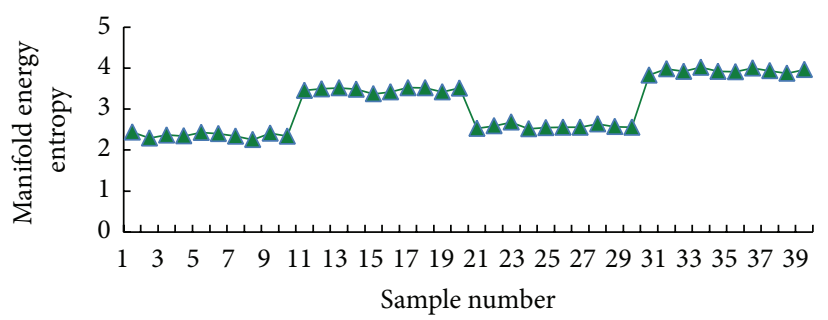

(a)

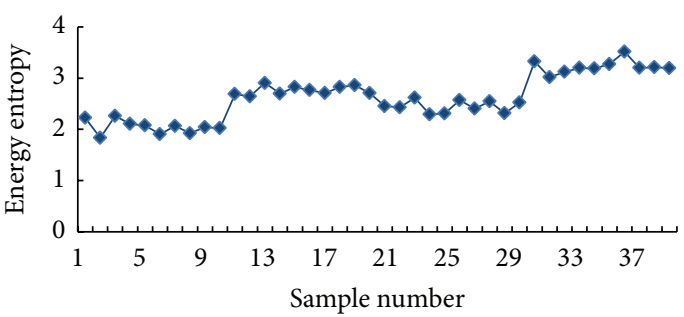

(b)

FIGURE 9: (a) Manifold energy entropy of the four fault signals and (b) energy entropy of the four fault signals.

the energy entropy shown in Figure 9(b) cannot provide a clear distinction between inner-race faults and ball faults. Thus, the manifold energy entropy is more appropriate for classifying rolling-bearing faults.

4.3.2. Manifold Energy Correlation Coefficient. The manifold energy correlation coefficient (manifold energy corcoef $(i)$ for short) is obtained by calculating the manifold energy corcoef $(i)$ between $\mathbf{E}_{f 1}, \mathbf{E}_{f 2}, \ldots, \mathbf{E}_{f 6}$ and $\mathbf{E}_{t}$. The results are shown in Figure 10.

As shown in Figure 10, the manifold energy corcoef $(i)$ can generally distinguish different fault signals, but different manifold energies corcoef $(i)$ have different abilities. First, corcoef(1) can generally distinguish four rolling-bearing failures. $\operatorname{corcoef}(2)$ is also suitable for distinguishing failures, except for the normal and ball fault samples. corcoef(3) failed to distinguish the ball fault and outer-race fault, $\operatorname{corcoef}(4)$ failed to distinguish the inner-race fault and ball fault, and corcoef(5) and corcoef(6) failed to distinguish all faults. Thus, corcoef(1) is accepted as the parameter that is best able to distinguish different rolling-bearing failures.

Figure 11 presents the energy correlation coefficient (hereafter denoted as "energy corcoef $(i)$ "), which is calculated using six large energy bands of the HHT time-frequency energy histogram without manifold analysis. As shown in Figure 11, the energy $\operatorname{corcoef}(i)$, where $i=1, \ldots, 6$, cannot provide clear distinctions and thus is not suitable for classifying different rolling-bearing faults.

4.3.3. Manifold Energy Sparsity. The energy distributions of different fault signals are different, as are the energy distributions of different regions in the time-frequency energy histogram. Figure 12(a) presents the manifold energy sparsity of four rolling-bearing signals. As shown in Figure 12(a), the manifold energy sparsity can effectively distinguish different fault samples and can be used to classify different rollingbearing faults. Figure 12(b) presents the energy sparsity of four types of signal samples. Although the energy sparsity can distinguish different rolling-bearing samples, the energy sparsity within the sample fluctuations, and the difference in energy sparsity of the inter-class sample is not obvious. Therefore, the energy sparsity is not a rolling-bearing fault parameter.

4.3.4. Manifold Energy Mutual Information. We divide the tensor manifold time-frequency energy histogram and HHT time-frequency energy histogram of the four types of signal 

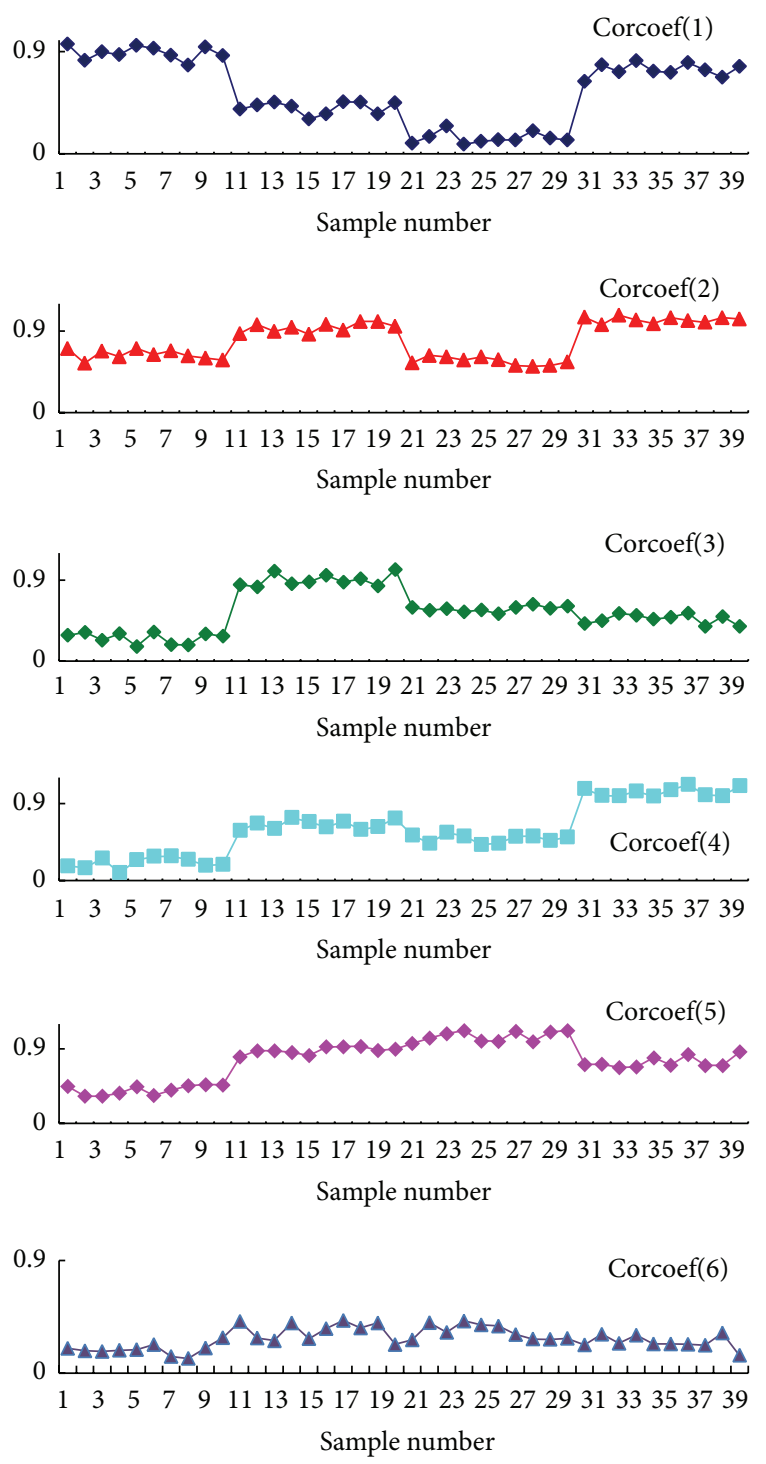

FIGURE 10: Manifold energy correlation coefficient of the four fault signals.

samples into 6 regions based on the frequency. Then, we calculate the corresponding mutual manifold energy information and mutual energy information.

As described in Figure 13(a), different rolling-bearing faults can be accurately distinguished using the mutual manifold energy information, which is clearly different among the fault samples; thus, the mutual manifold energy information can be used as the rolling-bearing fault characteristic parameter. Figure 13(b) illustrates that the mutual energy information of normal and ball fault samples is similar, and, thus, these two fault types cannot be distinguished. Therefore, the energy mutual information is not suitable for use as the rolling-bearing fault characteristic parameter.

4.3.5. Manifold Energy Kurtosis. We calculate the manifold energy kurtosis and energy kurtosis based on the corresponding energy histograms. Figure 14(a) presents the manifold
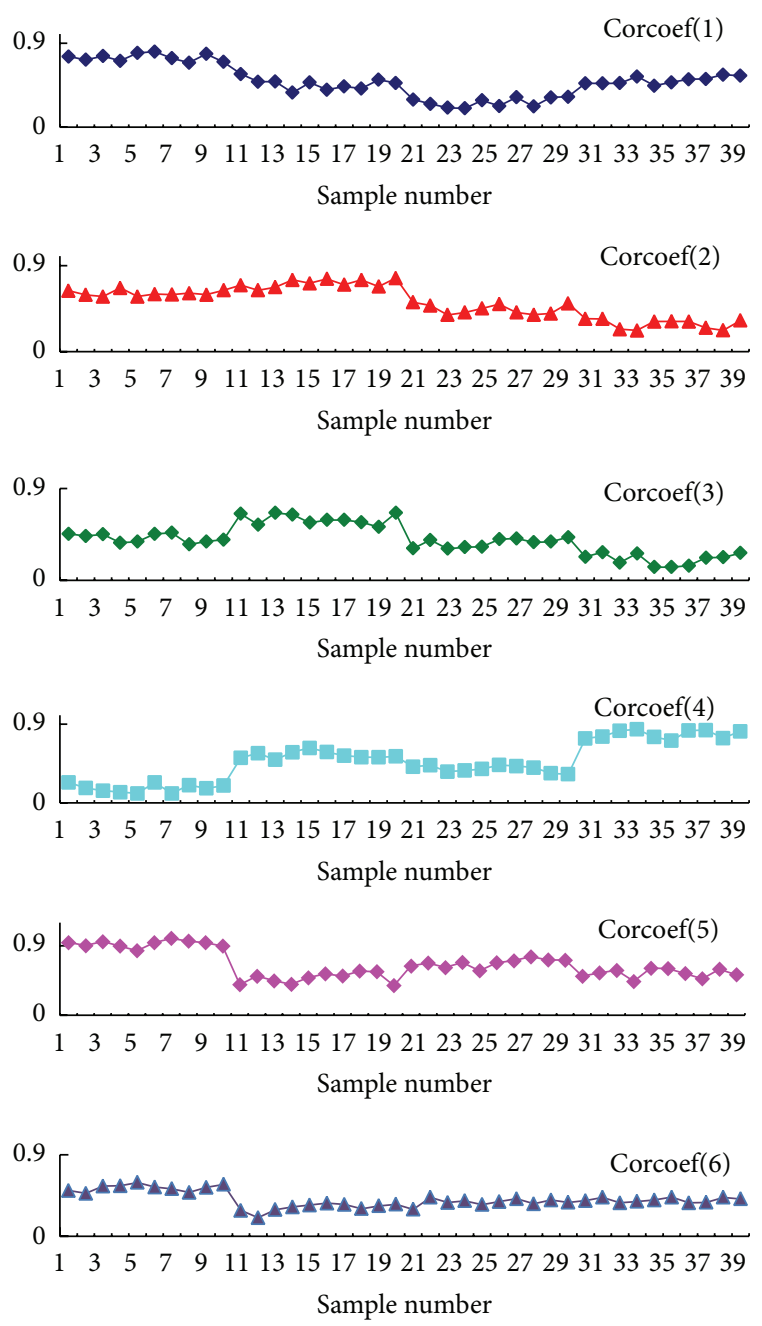

FIGURE 11: Energy correlation coefficient of the four fault signals.

energy kurtosis of the four different samples; the manifold energy kurtosis varies significantly, and, thus, the mutual energy manifold information can be used to classify different rolling-bearing faults. Figure 14(b) presents the energy kurtosis of the four different samples; as shown, all samples exhibit highly similar energy kurtosis values. In particular, the values of the normal fault, ball fault, and outer-race fault are extremely similar. Therefore, we cannot distinguish different rolling-bearing faults using energy kurtosis.

4.4. Discussion. The merits of the proposed method for extracting the tensor manifold time-frequency characteristic parameters are mainly based on the fact that the tensor manifold time-frequency feature explores the time-varying characteristic of the nonstationary fault signals. The tensor manifold utilizes the HHT time-frequency fault feature of the rolling-bearing fault vibration signals. Thus, the advanced feature is suitable for nonstationary vibration signals. Moreover, there are simple features that are widely used for classification in rolling-bearing fault diagnosis, such as timedomain features (e.g., kurtosis, variance), frequency-domain 


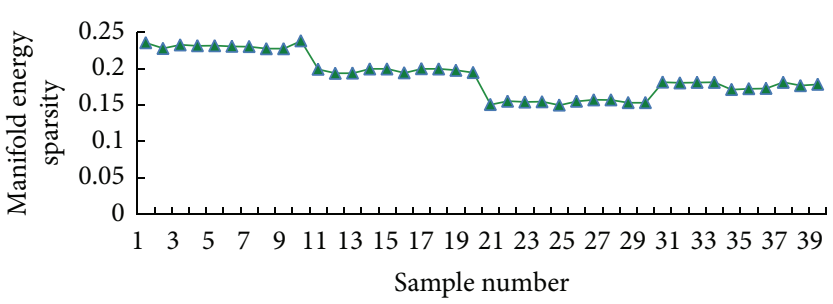

(a)

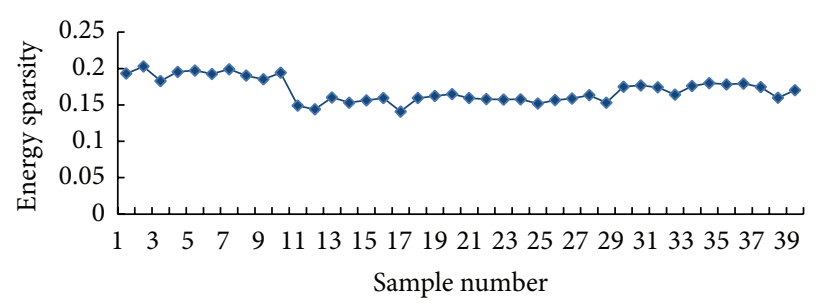

(b)

FIGURE 12: (a) Manifold energy sparsity of the four fault signals and (b) energy sparsity of the four fault signals.

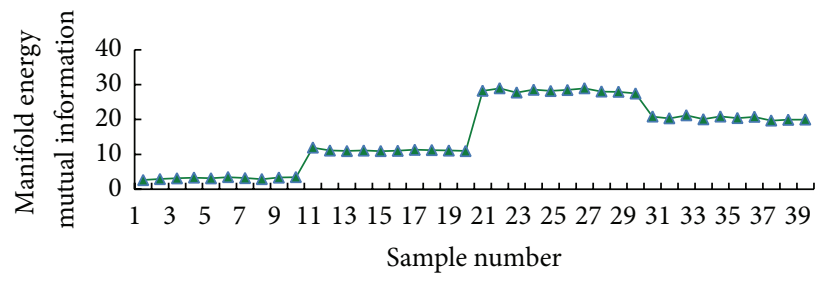

(a)

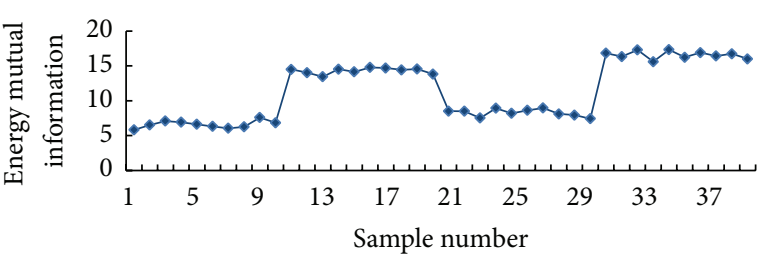

(b)

FIGURE 13: (a) Manifold energy mutual information of the four fault signals and (b) energy mutual information of the four fault signals.

features (e.g., subband energy), and time-frequency domain features (e.g., HHT time-frequency spectrum). These simple features are not as advantageous as the tensor manifold timefrequency features for capturing synthetic signal information. Thus, we use the tensor manifold time-frequency parameters for rolling-bearing fault diagnosis in this paper.

To demonstrate the benefit of the proposed parameters, simple features based on the HHT time-frequency spectrum are also conducted to analyze the four types of rolling-bearing fault signals. The test results are presented in Figures 9-13. These simple features perform worse in classification than do the tensor-manifold-based features. To avoid possible mistakes in pattern identification, it is necessary to improve the classification capability for reliable pattern diagnosis by exploring advanced features, which is the purpose of this paper.

\section{Rolling-Bearing Fault Classification}

The preceding analysis demonstrates that the five parameters (manifold energy entropy, manifold energy correlation coefficient, manifold energy sparsity, manifold energy mutual information, and manifold energy kurtosis) can efficiently distinguish the rolling-bearing fault states. Thus, they are used as the PNN input parameters for the bearing fault classification.

To verify the effectiveness of the manifold feature for identifying the four bearing faults, 20 samples of four types (normal, inner-race faults, ball faults, and outer-race faults) were used as training samples. The other 20 samples of each type were used for classification purposes. Each sample was extracted for the five aforementioned manifold feature parameters. The characteristic parameters of 80 training samples were used to train the PNN, and the numbers of nodes in the four PNNs were 5, 30, 4, and 4. Finally, the characteristic parameters of the 80 to-be-classified samples were input into the PNN for classification. The PNN classification results of the four bearing faults are shown in Table 1.

Table 1 illustrates that, when the tensor manifold timefrequency characteristic parameters are used as inputs for the PNN, four types of rolling-bearing fault samples can be effectively distinguished and each of the 20 to-be-classified samples for each type of fault can be correctly classified. The normal sample classification exhibits the best results, whereas the minimum components of the category vectors of the inner-race fault samples, ball fault samples, and outerrace fault samples are $0.92,0.93$, and 0.92 , respectively. The classification results of the PNN indicate that the rollingbearing fault condition can be effectively described using the tensor manifold time-frequency characteristic parameters and that the rolling-bearing fault type can be accurately identified with the PNN.

To compare the proposed method with traditional extraction methods, we extract the five defined parameters of the same training and to-be-classified samples using the traditional HHT time-frequency method as the PNN input parameters for the bearing fault classification. The results are shown in Table 2.

Table 2 illustrates that, when the HHT time-frequency characteristic parameters are used as inputs to the PNN, four types of rolling-bearing fault samples can generally be distinguished, but the distinction is not adequate. The normal sample classification exhibits the best results, whereas the minimum components of the category vector of the innerrace fault samples, ball fault samples, and outer-race faults are $0.69,0.76$, and 0.73 , respectively.

In Table 1, the minimum components of the category vectors of the four bearing faults are 0.92 . In contrast, in Table 2, except the normal-state, the components of the 


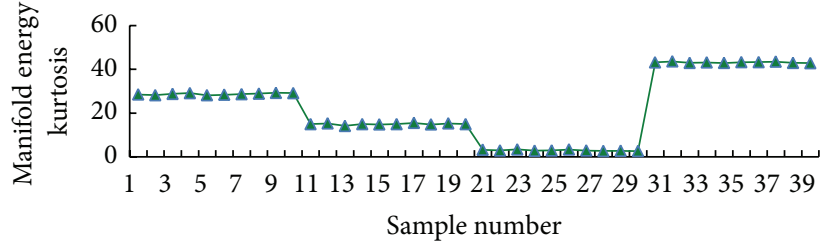

(a)

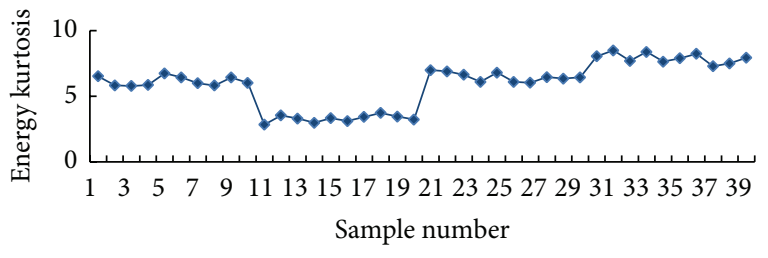

(b)

FIGURE 14: (a) Manifold energy kurtosis of the four fault signals and (b) energy kurtosis of the four fault signals.

TABLE 1: Classification results of the four bearing faults using the PNN with the proposed tensor manifold features.

\begin{tabular}{|c|c|c|c|c|c|c|c|c|c|c|c|c|c|c|c|c|}
\hline \multirow{2}{*}{$\begin{array}{l}\text { Sample } \\
1\end{array}$} & \multicolumn{4}{|c|}{$\begin{array}{l}\text { Category vector of the } \\
\text { normal-state samples }\end{array}$} & \multicolumn{4}{|c|}{$\begin{array}{l}\text { Category vector of the } \\
\text { inner-race fault samples }\end{array}$} & \multicolumn{4}{|c|}{$\begin{array}{l}\text { Category vector of the } \\
\text { ball fault samples }\end{array}$} & \multicolumn{4}{|c|}{$\begin{array}{l}\text { Category vector of the } \\
\text { outer-race fault samples }\end{array}$} \\
\hline & 1 & 0 & 0 & 0 & 0 & 0.97 & 0.01 & 0.02 & 0 & 0.01 & 0.99 & 0 & 0 & 0.01 & 0.02 & 0.97 \\
\hline 2 & 1 & 0 & 0 & 0 & 0 & 0.99 & 0 & 0.01 & 0 & 0.02 & 0.97 & 0.01 & 0 & 0.05 & 0.03 & 0.92 \\
\hline 3 & 1 & 0 & 0 & 0 & 0 & 0.95 & 0.03 & 0.02 & 0 & 0.02 & 0.97 & 0.01 & 0 & 0.03 & 0.02 & 0.95 \\
\hline 4 & 1 & 0 & 0 & 0 & 0 & 0.98 & 0.01 & 0.01 & 0 & 0.03 & 0.95 & 0.02 & 0 & 0.02 & 0.05 & 0.93 \\
\hline 5 & 1 & 0 & 0 & 0 & 0 & 0.93 & 0.05 & 0.02 & 0 & 0.02 & 0.98 & 0 & 0 & 0.02 & 0.02 & 0.96 \\
\hline 6 & 1 & 0 & 0 & 0 & 0 & 0.97 & 0.02 & 0.01 & 0 & 0.01 & 0.96 & 0.03 & 0 & 0.05 & 0.03 & 0.92 \\
\hline 7 & 1 & 0 & 0 & 0 & 0 & 0.95 & 0.02 & 0.03 & 0 & 0.02 & 0.97 & 0.01 & 0 & 0.02 & 0.01 & 0.97 \\
\hline 8 & 1 & 0 & 0 & 0 & 0 & 0.92 & 0.03 & 0.05 & 0 & 0.03 & 0.93 & 0.04 & 0 & 0.04 & 0.03 & 0.93 \\
\hline 9 & 1 & 0 & 0 & 0 & 0 & 0.94 & 0.02 & 0.04 & 0 & 0.02 & 0.95 & 0.03 & 0 & 0.03 & 0.02 & 0.95 \\
\hline 10 & 1 & 0 & 0 & 0 & 0 & 0.96 & 0.02 & 0.02 & 0 & 0.01 & 0.97 & 0.02 & 0 & 0.01 & 0.01 & 0.98 \\
\hline 11 & 1 & 0 & 0 & 0 & 0 & 0.93 & 0.04 & 0.03 & 0 & 0.01 & 0.98 & 0.01 & 0 & 0.03 & 0.01 & 0.96 \\
\hline 12 & 1 & 0 & 0 & 0 & 0 & 0.96 & 0.03 & 0.01 & 0 & 0.01 & 0.99 & 0 & 0 & 0.02 & 0.03 & 0.95 \\
\hline 13 & 1 & 0 & 0 & 0 & 0 & 0.97 & 0.01 & 0.02 & 0 & 0.02 & 0.96 & 0.03 & 0 & 0.01 & 0.02 & 0.97 \\
\hline 14 & 1 & 0 & 0 & 0 & 0 & 0.97 & 0.01 & 0.02 & 0 & 0.02 & 0.97 & 0.01 & 0 & 0.01 & 0.03 & 0.96 \\
\hline 15 & 1 & 0 & 0 & 0 & 0 & 0.95 & 0.03 & 0.02 & 0 & 0.02 & 0.95 & 0.03 & 0 & 0.06 & 0.02 & 0.92 \\
\hline 16 & 1 & 0 & 0 & 0 & 0 & 0.98 & 0.01 & 0.01 & 0 & 0 & 0.98 & 0.02 & 0 & 0.03 & 0.04 & 0.93 \\
\hline 17 & 1 & 0 & 0 & 0 & 0 & 0.96 & 0.01 & 0.03 & 0 & 0.03 & 0.96 & 0.01 & 0 & 0.03 & 0.02 & 0.95 \\
\hline 18 & 1 & 0 & 0 & 0 & 0 & 0.97 & 0.02 & 0.01 & 0 & 0.01 & 0.97 & 0.02 & 0 & 0.03 & 0.01 & 0.96 \\
\hline 19 & 1 & 0 & 0 & 0 & 0 & 0.93 & 0.03 & 0.04 & 0 & 0.02 & 0.96 & 0.02 & 0 & 0.03 & 0.04 & 0.93 \\
\hline 20 & 1 & 0 & 0 & 0 & 0 & 0.96 & 0.01 & 0.03 & 0 & 0.03 & 0.93 & 0.04 & 0 & 0.03 & 0.05 & 0.92 \\
\hline
\end{tabular}

category vectors of the inner-race fault, the ball fault, and the outer-race fault above 0.85 account for $65 \%, 80 \%$, and $60 \%$, respectively. Compared with the results of the PNN, which uses tensor manifold time-frequency characteristic parameters as inputs, the classification performance with traditional HHT time-frequency features is relatively poor.

To verify the effectiveness of the manifold feature for identifying different damage degrees in the same fault type of rolling-bearing status, the proposed method was used to classify the inner-race fault samples with different damage degrees. 20 samples of four degrees (normal, mild damage, moderate damage, and severe damage) were used as training samples. The other 10 samples of each degree were used for classification purposes. The PNN classification results of the to-be-classified inner-race fault samples are shown in Table 3.

To compare the proposed method with traditional extraction methods, we extract the five defined parameters of the same training and to-be-classified inner-race fault samples with different damage degrees using the traditional HHT time-frequency method as the PNN input parameters for the bearing fault classification. The results are shown in Table 4.

Table 4 depicts that, when the HHT time-frequency characteristic parameters are used as inputs to the PNN, inner-race fault samples with different damage degrees can generally be distinguished, but the distinction is not adequate. The minimum components of the category vector of the inner-race mild-damage fault samples, moderate-damage fault samples, and severe-damage fault samples are $0.83,0.86$, and 0.78 , respectively.

In Table 3, the minimum components of the category vectors of the four bearing faults are 0.93 . In contrast, in Table 4, except the normal-state, the components of the category vectors of the inner-race fault, the ball fault, and the outer-race fault above 0.85 account for $90 \%, 100 \%$, and $60 \%$, respectively. Compared with the results in Table 3, the results of the PNN, which uses the traditional HHT time-frequency characteristic parameters as inputs, indicate a relatively poor classification performance. 
TABLE 2: Classification results of the four bearing faults using the PNN with traditional HHT time-frequency features.

\begin{tabular}{|c|c|c|c|c|c|c|c|c|c|c|c|c|c|c|c|c|}
\hline \multirow{2}{*}{$\begin{array}{l}\text { Sample } \\
1\end{array}$} & \multicolumn{4}{|c|}{$\begin{array}{l}\text { Category vector of the } \\
\text { normal-state samples }\end{array}$} & \multicolumn{4}{|c|}{$\begin{array}{l}\text { Category vector of the } \\
\text { inner-race fault samples }\end{array}$} & \multicolumn{4}{|c|}{$\begin{array}{l}\text { Category vector of the } \\
\text { ball fault samples }\end{array}$} & \multicolumn{4}{|c|}{$\begin{array}{l}\text { Category vector of the } \\
\text { outer-race fault samples }\end{array}$} \\
\hline & 1 & 0 & 0 & 0 & 0 & 0.90 & 0.05 & 0.05 & 0 & 0.10 & 0.89 & 0.01 & 0 & 0.06 & 0.07 & 0.83 \\
\hline 2 & 1 & 0 & 0 & 0 & 0 & 0.92 & 0.03 & 0.05 & 0 & 0.12 & 0.87 & 0.01 & 0 & 0.05 & 0.06 & 0.89 \\
\hline 3 & 1 & 0 & 0 & 0 & 0 & 0.89 & 0.05 & 0.06 & 0 & 0.12 & 0.79 & 0.09 & 0 & 0.07 & 0.08 & 0.85 \\
\hline 4 & 1 & 0 & 0 & 0 & 0 & 0.90 & 0.06 & 0.04 & 0 & 0.13 & 0.85 & 0.12 & 0 & 0.05 & 0.04 & 0.91 \\
\hline 5 & 1 & 0 & 0 & 0 & 0 & 0.87 & 0.06 & 0.07 & 0 & 0.10 & 0.78 & 0.12 & 0 & 0.06 & 0.08 & 0.86 \\
\hline 6 & 1 & 0 & 0 & 0 & 0 & 0.92 & 0.04 & 0.08 & 0 & 0.13 & 0.76 & 0.11 & 0 & 0.15 & 0.06 & 0.79 \\
\hline 7 & 1 & 0 & 0 & 0 & 0 & 0.91 & 0.05 & 0.04 & 0 & 0.07 & 0.87 & 0.06 & 0 & 0.06 & 0.07 & 0.87 \\
\hline 8 & 1 & 0 & 0 & 0 & 0 & 0.82 & 0.08 & 0.1 & 0 & 0.02 & 0.93 & 0.05 & 0 & 0.15 & 0.12 & 0.73 \\
\hline 9 & 1 & 0 & 0 & 0 & 0 & 0.87 & 0.08 & 0.05 & 0 & 0.08 & 0.85 & 0.07 & 0 & 0.07 & 0.08 & 0.75 \\
\hline 10 & 1 & 0 & 0 & 0 & 0 & 0.93 & 0.05 & 0.02 & 0 & 0.07 & 0.87 & 0.06 & 0 & 0.05 & 0.07 & 0.88 \\
\hline 11 & 1 & 0 & 0 & 0 & 0 & 0.69 & 0.14 & 0.17 & 0 & 0.01 & 0.88 & 0.11 & 0 & 0.06 & 0.08 & 0.86 \\
\hline 12 & 1 & 0 & 0 & 0 & 0 & 0.74 & 0.12 & 0.14 & 0 & 0.20 & 0.79 & 0.01 & 0 & 0.07 & 0.08 & 0.85 \\
\hline 13 & 1 & 0 & 0 & 0 & 0 & 0.80 & 0.12 & 0.08 & 0 & 0.08 & 0.88 & 0.04 & 0 & 0.03 & 0.06 & 0.91 \\
\hline 14 & 1 & 0 & 0 & 0 & 0 & 0.86 & 0.09 & 0.05 & 0 & 0.07 & 0.87 & 0.06 & 0 & 0.07 & 0.04 & 0.89 \\
\hline 15 & 1 & 0 & 0 & 0 & 0 & 0.75 & 0.23 & 0.02 & 0 & 0.06 & 0.89 & 0.05 & 0 & 0.08 & 0.09 & 0.83 \\
\hline 16 & 1 & 0 & 0 & 0 & 0 & 0.78 & 0.10 & 0.12 & 0 & 0.03 & 0.93 & 0.02 & 0 & 0.09 & 0.10 & 0.81 \\
\hline 17 & 1 & 0 & 0 & 0 & 0 & 0.86 & 0.12 & 0.12 & 0 & 0.06 & 0.87 & 0.07 & 0 & 0.07 & 0.04 & 0.89 \\
\hline 18 & 1 & 0 & 0 & 0 & 0 & 0.87 & 0.06 & 0.07 & 0 & 0.09 & 0.89 & 0.02 & 0 & 0.03 & 0.08 & 0.89 \\
\hline 19 & 1 & 0 & 0 & 0 & 0 & 0.83 & 0.13 & 0.14 & 0 & 0.02 & 0.91 & 0.07 & 0 & 0.07 & 0.01 & 0.83 \\
\hline 20 & 1 & 0 & 0 & 0 & 0 & 0.92 & 0.05 & 0.03 & 0 & 0.05 & 0.92 & 0.03 & 0 & 0.07 & 0.11 & 0.82 \\
\hline
\end{tabular}

TABLE 3: Classification results of the inner-race fault using the PNN with the proposed tensor manifold features.

\begin{tabular}{|c|c|c|c|c|c|c|c|c|c|c|c|c|c|c|c|c|}
\hline \multirow{2}{*}{$\begin{array}{l}\text { Sample } \\
1\end{array}$} & \multicolumn{4}{|c|}{$\begin{array}{l}\text { Category vector of the } \\
\text { normal-state samples }\end{array}$} & \multicolumn{4}{|c|}{$\begin{array}{l}\text { Category vector of the } \\
\text { inner-race } \\
\text { mild-damage } \\
\text { fault samples }\end{array}$} & \multicolumn{4}{|c|}{$\begin{array}{l}\text { Category vector of the } \\
\text { inner-race } \\
\text { moderate-damage } \\
\text { fault samples }\end{array}$} & \multicolumn{4}{|c|}{$\begin{array}{l}\text { Category vector of the } \\
\text { inner-race } \\
\text { severe-damage } \\
\text { fault samples }\end{array}$} \\
\hline & 1 & 0 & 0 & 0 & 0 & 0.97 & 0.01 & 0.02 & 0 & 0.01 & 0.98 & 0.01 & 0 & 0.01 & 0.02 & 0.97 \\
\hline 2 & 1 & 0 & 0 & 0 & 0 & 0.96 & 0.02 & 0.02 & 0 & 0.02 & 0.97 & 0.01 & 0 & 0.02 & 0.02 & 0.96 \\
\hline 3 & 1 & 0 & 0 & 0 & 0 & 0.98 & 0.01 & 0.01 & 0 & 0.01 & 0.97 & 0.02 & 0 & 0.03 & 0.02 & 0.95 \\
\hline 4 & 1 & 0 & 0 & 0 & 0 & 0.95 & 0.02 & 0.03 & 0 & 0.03 & 0.95 & 0.02 & 0 & 0.02 & 0.02 & 0.96 \\
\hline 5 & 1 & 0 & 0 & 0 & 0 & 0.96 & 0.02 & 0.02 & 0 & 0.01 & 0.98 & 0.01 & 0 & 0.01 & 0.03 & 0.96 \\
\hline 6 & 1 & 0 & 0 & 0 & 0 & 0.97 & 0.01 & 0.02 & 0 & 0.01 & 0.96 & 0.03 & 0 & 0.05 & 0.02 & 0.93 \\
\hline 7 & 1 & 0 & 0 & 0 & 0 & 0.94 & 0.05 & 0.02 & 0 & 0.02 & 0.95 & 0.03 & 0 & 0.03 & 0.02 & 0.95 \\
\hline 8 & 1 & 0 & 0 & 0 & 0 & 0.95 & 0.03 & 0.02 & 0 & 0.02 & 0.97 & 0.01 & 0 & 0.01 & 0.01 & 0.98 \\
\hline 9 & 1 & 0 & 0 & 0 & 0 & 0.94 & 0.02 & 0.04 & 0 & 0.04 & 0.94 & 0.02 & 0 & 0.03 & 0.03 & 0.94 \\
\hline 10 & 1 & 0 & 0 & 0 & 0 & 0.93 & 0.02 & 0.05 & 0 & 0.04 & 0.93 & 0.03 & 0 & 0.03 & 0.01 & 0.96 \\
\hline
\end{tabular}

\section{Conclusion}

This paper studies the problem of rolling-bearing fault feature extraction. A time-frequency feature extraction method based on tensor manifolds for rolling bearings was proposed to overcome the deficiencies of the traditional HHT timefrequency feature extraction methods and to remove redundant time-frequency feature information. The HHT timefrequency energy histograms of the rolling-bearing fault signal were used to compose high-dimensional time-frequency fault feature sets. On this basis, the signal time-frequency characteristics were extracted using tensor manifold learning. Five tensor manifold time-frequency characteristic parameters were defined: manifold energy entropy, manifold energy correlation coefficient, manifold energy sparsity, manifold energy mutual information, and manifold energy kurtosis. These characteristic parameters and a PNN were combined to accurately classify rolling-bearing fault samples. The tensor manifold method can realize the nonlinear fusion of the time-frequency information, which can effectively extract the intrinsic nonlinear characteristics of high-dimensional timefrequency combination, and avoid the loss of information 
TABLE 4: Classification results of the inner-race fault using the PNN with traditional HHT time-frequency features.

\begin{tabular}{|c|c|c|c|c|c|c|c|c|c|c|c|c|c|c|c|c|}
\hline \multirow{2}{*}{$\begin{array}{l}\text { Sample } \\
1\end{array}$} & \multicolumn{4}{|c|}{$\begin{array}{l}\text { Category vector of the } \\
\text { normal-state samples }\end{array}$} & \multicolumn{4}{|c|}{$\begin{array}{l}\text { Category vector of the } \\
\text { inner-race } \\
\text { mild-damage } \\
\text { fault samples }\end{array}$} & \multicolumn{4}{|c|}{$\begin{array}{l}\text { Category vector of the } \\
\text { inner-race } \\
\text { moderate-damage } \\
\text { fault samples }\end{array}$} & \multicolumn{4}{|c|}{$\begin{array}{l}\text { Category vector of the } \\
\text { inner-race } \\
\text { severe-damage } \\
\text { fault samples }\end{array}$} \\
\hline & 1 & 0 & 0 & 0 & 0 & 0.92 & 0.05 & 0.03 & 0 & 0.11 & 0.87 & 0.02 & 0 & 0.12 & 0.05 & 0.83 \\
\hline 2 & 1 & 0 & 0 & 0 & 0 & 0.87 & 0.06 & 0.07 & 0 & 0.06 & 0.89 & 0.05 & 0 & 0.05 & 0.06 & 0.89 \\
\hline 3 & 1 & 0 & 0 & 0 & 0 & 0.88 & 0.06 & 0.06 & 0 & 0.07 & 0.91 & 0.02 & 0 & 0.06 & 0.06 & 0.88 \\
\hline 4 & 1 & 0 & 0 & 0 & 0 & 0.91 & 0.07 & 0.02 & 0 & 0.03 & 0.92 & 0.05 & 0 & 0.06 & 0.02 & 0.92 \\
\hline 5 & 1 & 0 & 0 & 0 & 0 & 0.83 & 0.09 & 0.08 & 0 & 0.06 & 0.90 & 0.04 & 0 & 0.03 & 0.06 & 0.91 \\
\hline 6 & 1 & 0 & 0 & 0 & 0 & 0.89 & 0.05 & 0.06 & 0 & 0.09 & 0.86 & 0.05 & 0 & 0.05 & 0.06 & 0.89 \\
\hline 7 & 1 & 0 & 0 & 0 & 0 & 0.92 & 0.04 & 0.04 & 0 & 0.05 & 0.91 & 0.04 & 0 & 0.06 & 0.03 & 0.91 \\
\hline 8 & 1 & 0 & 0 & 0 & 0 & 0.90 & 0.06 & 0.04 & 0 & 0.03 & 0.92 & 0.05 & 0 & 0.05 & 0.12 & 0.83 \\
\hline 9 & 1 & 0 & 0 & 0 & 0 & 0.91 & 0.04 & 0.05 & 0 & 0.09 & 0.86 & 0.05 & 0 & 0.15 & 0.07 & 0.78 \\
\hline 10 & 1 & 0 & 0 & 0 & 0 & 0.92 & 0.03 & 0.05 & 0 & 0.05 & 0.89 & 0.06 & 0 & 0.11 & 0.06 & 0.83 \\
\hline
\end{tabular}

caused by traditional manifold-learning methods. Compared with the HHT time-frequency characteristic parameters, the tensor manifold time-frequency characteristic parameters can more effectively distinguish the four bearing faults, different damage degrees in the same fault type, and different fault types with different damage degrees because of its strong nonlinearity and reduced information redundancy. The effectiveness of the proposed method was verified using real rolling-bearing fault signals. Thus, this paper provides an important method to solve the rolling-bearing feature extraction problems.

\section{Conflict of Interests}

The authors declare that there is no conflict of interests regarding the publication of this paper.

\section{Acknowledgments}

This work is supported by the National Natural Science Foundation of China (no. 51375067), the Aviation Science Foundation of China (no. 20132163010), and the Fundamental Research Funds for the Central Universities of China (no. DUT13JS08).

\section{References}

[1] Q. Liu, F. Chen, Z. Zhou, and Q. Wei, "Fault diagnosis of rolling bearing based on wavelet package transform and ensemble empirical mode decomposition," Advances in Mechanical Engineering, vol. 2013, Article ID 792584, 6 pages, 2013.

[2] X. S. Lou and K. A. Loparo, "Bearing fault diagnosis based on wavelet transform and fuzzy inference," Mechanical Systems and Signal Processing, vol. 18, no. 5, pp. 1077-1095, 2004.

[3] F. Cong, J. Chen, G. Dong, and M. Pecht, "Vibration model of rolling element bearings in a rotor-bearing system for fault diagnosis," Journal of Sound and Vibration, vol. 332, no. 8, pp. 2081-2097, 2013.

[4] L. Jiang, J. Xuan, and T. Shi, "Feature extraction based on semisupervised kernel Marginal Fisher analysis and its application in bearing fault diagnosis," Mechanical Systems and Signal Processing, vol. 41, no. 1-2, pp. 113-126, 2013.

[5] Y. Lei, Z. He, and Y. Zi, "A new approach to intelligent fault diagnosis of rotating machinery," Expert Systems with Applications, vol. 35, no. 4, pp. 1593-1600, 2008.

[6] Z. K. Peng, P. W. Tse, and F. L. Chu, "A comparison study of improved Hilbert-Huang transform and wavelet transform: application to fault diagnosis for rolling bearing," Mechanical Systems and Signal Processing, vol. 19, no. 5, pp. 974-988, 2005.

[7] A. Papandreou-Suppappola, Applications in TimeFrequency Signal Processing, vol. 10, CRC Press, Boca Raton, Fla, USA, 2003.

[8] B. Boashash, Time Frequency Signal Analysis and Processing, Prentice Hall, New York, NY, USA, 2002.

[9] K. Gröchenig, Foundations of Time-Frequency Analysis, Birkhauser, Boston, Mass, USA, 2000.

[10] S. Stanković, "Time-frequency analysis and its application in digital watermarking," Eurasip Journal on Advances in Signal Processing, vol. 2010, Article ID 579295, 2010.

[11] Z. W. Wang and C. Y. Hu, "Shock spectra and damage boundary curves for nonlinear package cushioning system," Packaging Technology and Science, vol. 12, no. 5, pp. 207-217, 1999.

[12] J. Y. Zhang, Y. Y. Zhang, and Y. B. Xie, "Applications of timefrequency analysis based on wavelet packet to incipient impulse faults diagnosis," Journal of Vibration Engineering, vol. 13, no. 2, pp. 66-72, 2000.

[13] L. M. Zhu, X. W. Niu, B. L. Zhong, and H. Ding, "Approach for extracting the time-frequency feature of a signal with application to machine condition monitoring," Journal of Vibration Engineering, vol. 17, no. 4, pp. 71-76, 2004.

[14] N. E. Huang, Z. Shen, S. R. Long et al., “The empirical mode decomposition and the Hilbert spectrum for nonlinear and non-stationary time series analysis," Proceedings of the Royal Society of London A, vol. 454, no. 1971, pp. 903-995, 1998.

[15] M. Gandetto, M. Guainazzo, and C. S. Regazzoni, "Use of timefrequency analysis and neural networks for mode identification in a wireless software-defined radio approach," EURASIP Journal on Advances in Signal Processing, vol. 2004, no. 12, pp. 17781790, 2004.

[16] Y. G. Lei, Z. J. He, and Y. Y. Zi, "EEMD method and WNN for fault diagnosis of locomotive roller bearings," Expert Systems with Applications, vol. 38, no. 6, pp. 7334-7341, 2011. 
[17] P. Shan and M. Li, "Nonlinear time-varying spectral analysis: HHT and MODWPT," Mathematical Problems in Engineering, vol. 2010, Article ID 618231, 14 pages, 2010.

[18] H. Li, P. Zhou, and Z. Zhang, "An investigation into machine pattern recognition based on time-frequency image feature extraction using a support vector machine," Journal of Mechanical Engineering Science, vol. 224, no. 4, pp. 981-994, 2010.

[19] H. K. Li, S. Zhou, and W. Z. Huang, "Time-frequency image feature extraction for machine condition classification and its application," Journal of Vibration and Shock, vol. 29, no. 7, pp. 184-188, 2010.

[20] Q. He, "Time-frequency manifold for nonlinear feature extraction in machinery fault diagnosis," Mechanical Systems and Signal Processing, vol. 35, no. 1-2, pp. 200-218, 2013.

[21] F. Dornaika and A. Assoum, "Enhanced and parameterless locality preserving projections for face recognition," Neurocomputing, vol. 99, pp. 448-457, 2013.

[22] M. Ravishankar and D. R. Rameshbabu, “Ten-LoPP: tensor locality preserving projections approach for moving object detection and tracking," in Proceedings of the 9th International Conference on Computing and InformationTechnology, pp. 291300, Springer, Berlin, Germany, 2013.

[23] "Bearings Vibration Data Set, Case Western Reserve University," http://csegroups.case.edu/bearingdatacenter/home. 


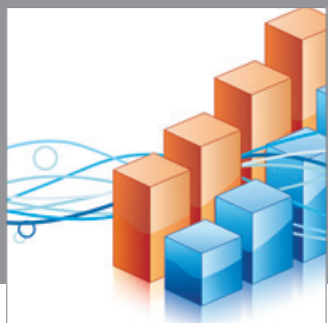

Advances in

Operations Research

mansans

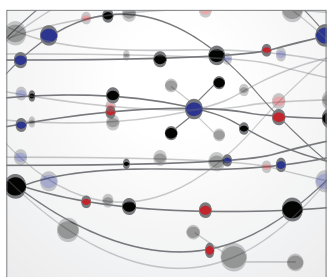

The Scientific World Journal
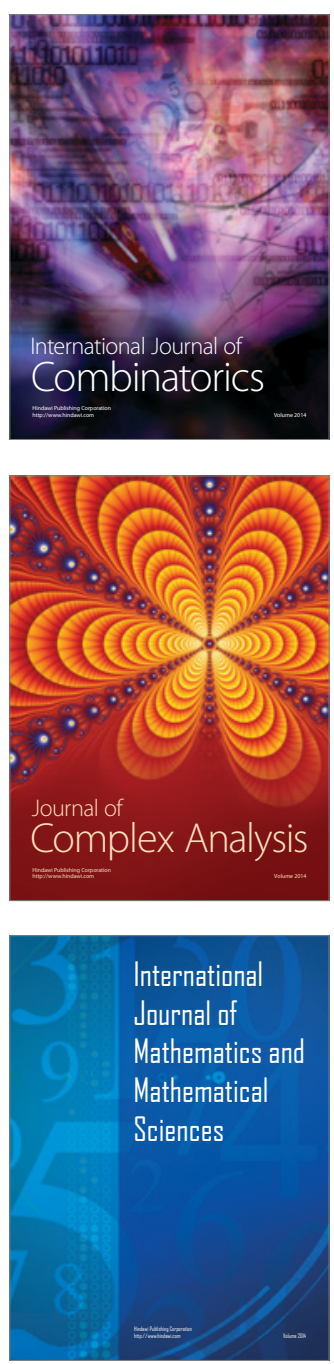
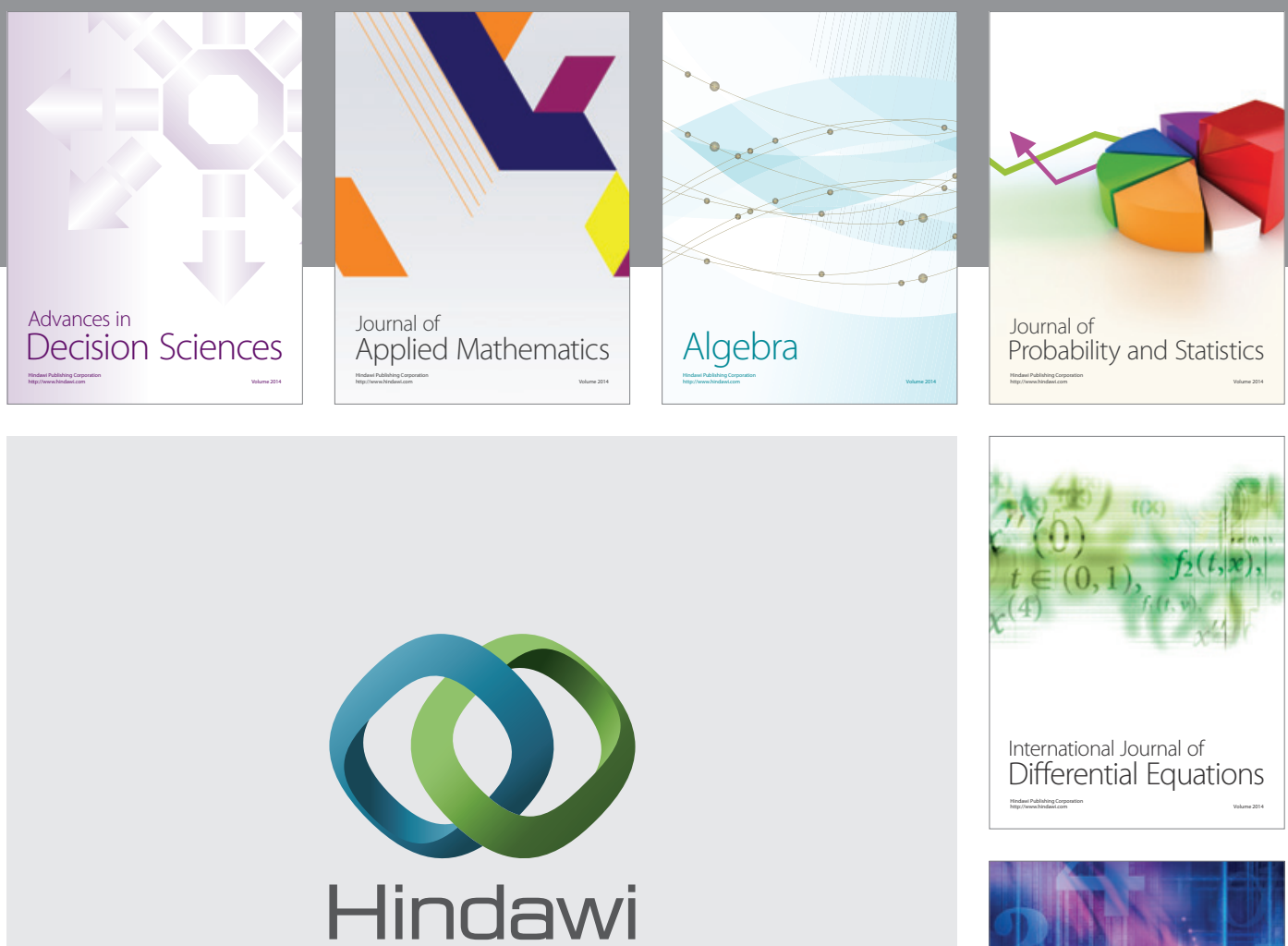

Submit your manuscripts at http://www.hindawi.com
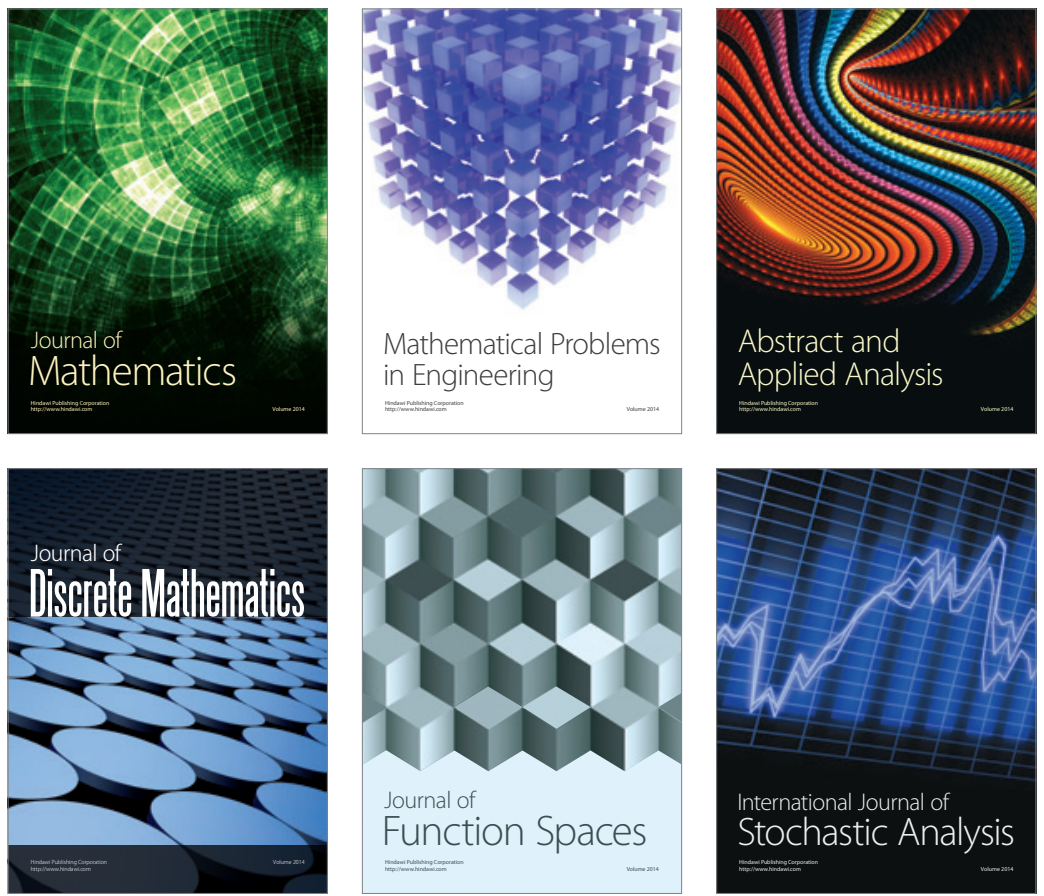

Journal of

Function Spaces

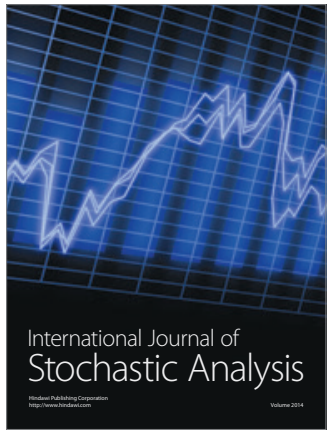

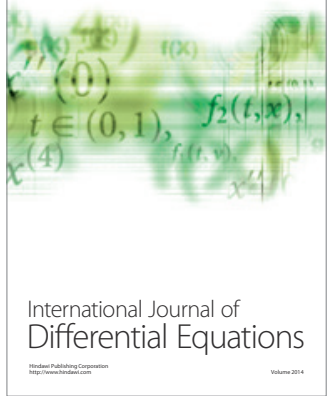
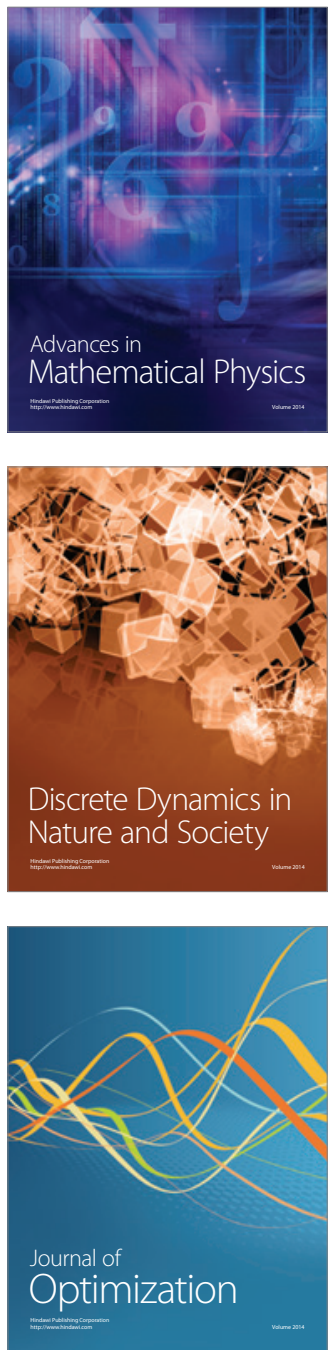